\title{
Two Models of Predator Decision Making under Uncertainty
}

\author{
by
}

Ian Dewan

\begin{abstract}
A thesis submitted to the
Faculty of Graduate and Postdoctoral Affairs

in partial fulfillment of the requirements for the degree of
\end{abstract}

\author{
Master of Science \\ in \\ Biology \\ Carleton University \\ Ottawa, Ontario
}

(C)2019, Ian Dewan 


\begin{abstract}
Predators are faced with an uncertain world. The presence of anti-predator defences, means that any potential prey item may actually be unpalatable, outright toxic, difficult to catch, or cause harm. In order to deal with this uncertainty regarding profitability, predators need strategies to make good decisions on what to attack based on the information about potential prey available to them. I develop two models of optimal decision making for predators. The first deals with generalizing from experience to novel prey types: I develop a Bayesian model framework that treats generalization as a process of learning about the distribution of prey in the environment, and apply it to a problem in generalization. The second deals with startle displays: I develop an extension of signal detection theory to cases where continued examination is possible, and apply it to predators faced with startle displays.
\end{abstract}




\section{ACKNOWLEDGEMENTS}

Firstly, I would like to thank my supervisor, Tom Sherratt for all his support and assistance over the course of my MSc. Every time I come out of a meeting with him, I feel better about the work I've done, and more optimistic about and better prepared for the next steps of my project. I've learned a great deal over the past two years, and I've had a great time doing it. I'm very grateful to have had the opportunity to work with Tom. I am also grateful to the other members of my advisory committee, Root Gorelick and Frithjof Lutscher, for their invaluable advice and assistance.

Working in the Sherratt lab has been a great experience, and a lot of fun, which is thanks largely to all of the great people I've met here over the past two years. Thank you to all the lab members, and in particular to Lauren Efford, Eric GuerraGrenier, and Sophie Potter. I'd like to especially thank Sophie, who has always been welcoming and supportive to everyone in the lab, and a great friend, and who also helped to select the name of the parameter $\Xi$.

I'd also like to thank Joey Beauveais-Feisthauer, Blair Drummond, and Luke Volk for many weeks of entertaining conversation and fun problems. Finally, I'd like to thank my family for always being there for me. 


\section{CONTENTS}

\begin{tabular}{ll}
\hline Abstract & ii
\end{tabular}

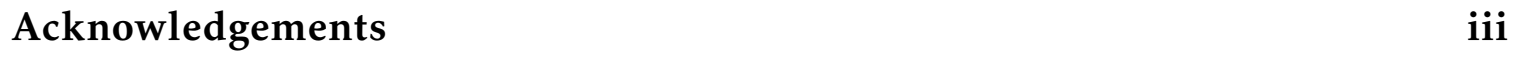

Contents $\quad$ iv

List of Tables $\quad$ vi

\begin{tabular}{|l|l}
\hline List of Figures & vii
\end{tabular}

$1 \quad$ Introduction: Anti-predator defences and predator decisions 1

2 Too close for comfort: Predator generalization of prey palatability 5

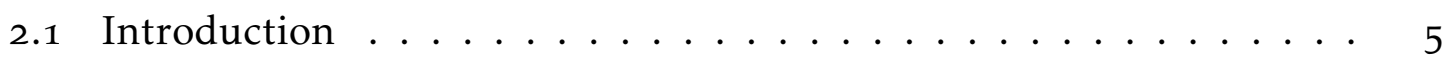

2.1 .1 Psychological aspects of generalization . . . . . . . . . 7

2.1 .2 Generalization and mimicry models. . . . . . . . . . . 9

2.2 Model framework . . . . . . . . . . . . . . 11

2.2 .1 Background $\ldots \ldots \ldots \ldots 11$

2.2 .2 The extended model . . . . . . . . . . . . 13

2.2 .3 Choosing priors .................... 14

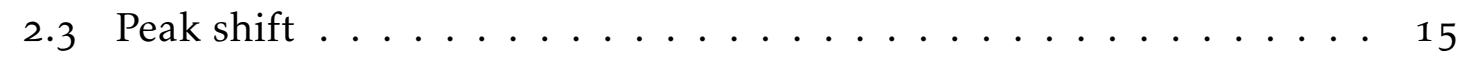

2.3 .1 A binary palatability model . . . . . . . . . . 17

2.3 .2 A second model .................. 21

2.4 A reconstruction of empirical results . . . . . . . . . . 23 


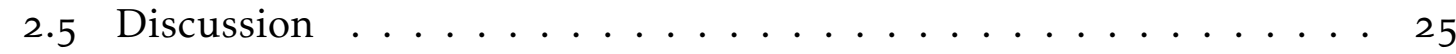

3 The Adaptive Advantage of Startling and Being Startled 31

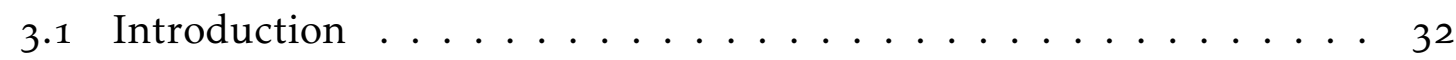

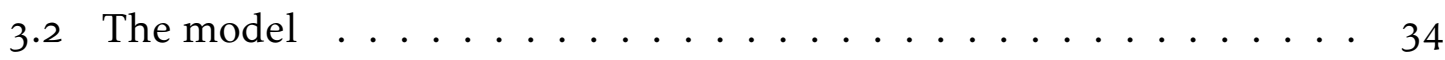

3.2.1 The expected reward ..................... 36

3.2 .2 Predicting future decisions . . . . . . . . . 38

3.2 .3 Existence of an optimum . . . . . . . . . . . 39

3.3 Results ............................... 40

3.4 A second model: distance . . . . . . . . . . . . 41

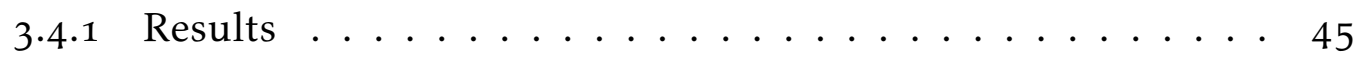

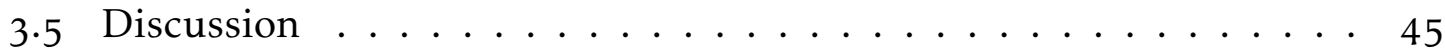

\begin{tabular}{|lll}
4 & Conclusion & 53
\end{tabular}

References $\quad 56$

\begin{tabular}{|lll}
\hline A Unnecessary extensions to the mixture model & 71
\end{tabular}

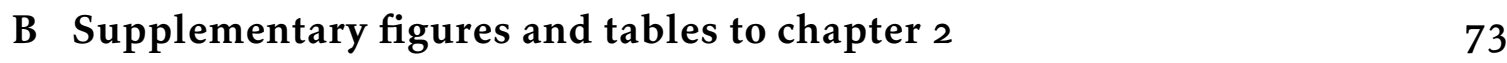

\begin{tabular}{lll}
\hline Upper bound on $n^{*}$ & 77
\end{tabular}

\begin{tabular}{|l|l|l|}
\hline S Supplementary figures to chapter 39 & 79
\end{tabular} 


\section{LIST OF TABLES}

2.1 Comparison of generalization in models of mimicry. . . . . . . . . 10

2.2 Degrees of peak shift from a binary palatability model. The peak loca-

tions and values are given for the palatable peak (the unpalatable peaks

can be extrapolated by symmetry). $n$ and $m$ are the assumed number of

populations for palatable and unpalatable prey respectively. The peak

shift is the distance between the peak location and the training example

phenotype, which was always $-1 . \ldots . \ldots . . \ldots 20$

B.1 Degrees of peak shift from a binary palatability model. As Table 2.2

but fixing $\sigma_{p}^{2} / \sigma_{w}^{2}=10$, and with the predator having made observations

at \pm the observation. . . . . . . . . . . . . . 76 


\section{LIST OF FIGURES}

2.1 Estimated palatabilities of a novel phenotype under a binary palatability model. $n$ and $m$ are the assumed number of populations of palatable and unpalatable prey respectively. The previous observations, palatable with phenotype -1 and unpalatable with phenotype 1 , are shown by the blue and red lines respectively. The phenotype is in units of $\sigma_{w}$. . . . . 19

2.2 Expected palatability for varying phenotypes. Coloured bands are the 95\% confidence intervals of the mean (solid line). The two solid lines correspond to models with mixtures of three and four normal distributions, respectively, and the two dashed lines to the locations of the previously observed palatable (blue) and unpalatable (red) prey. . . . . 22

2.3 The colour experiment of Baddeley et al. (2007); reproduced from Baddeley et al. (2007), Figure 2. Panel A shows the colour space simplex; the grey line indicates the locations of monochromatic light at the indicated wavelengths (nm). Panels B and C show the colours used in experiments 1 and 2 . The rewarded colour in training is indicated with $\mathrm{a}+$ in both panels. In panel $\mathrm{B}$, the numbered $\times$ show the respective unrewarded/test colours. In panel C, the unrewarded training colours are shown as $\bullet$, and novel colours as $\times$. . . . . . . . . . . . 24 
2.4 Results, real and simulated, of experiment 1. The upper panel (from Baddeley et al. (2007), Figure 3a) shows experimental generalization in groups 1 (top dotted), 2 (top solid), 3 (bottom solid), and 4 (bottom dotted). The lower panel shows simulated generalization, in single Dirichlet per type (Simple) and two Dirichlet per type (Double) versions. 26

2.5 Results, real and simulated, of experiment 2. The upper panel (from Baddeley et al. (2007), Figure 4) shows experimental generalization in groups 1 (dotted), and 2 (solid). The lower panel shows simulated generalization, in single Dirichlet per type (Simple) and two Dirichlet per type (Double) versions. . . . . . . . . . . . . . . . 27

3.1 The optimal action after having made a single observation of the prey, at a background frequency of desirable prey of $p=0.7$ and with $\Delta=$ $0.05=\Xi$. The optimal action is either to attack immediately (green area of each panel), flee immediately (red area), or continue observing for $n^{*}$ additional observations (coloured by $n^{*}$, as in legend). Columns correspond to values of $\sigma(0)=\sqrt{\sigma_{p}^{2}+\sigma_{e}^{2}}$, the standard deviation of a single observation, and rows to $r=\frac{\sigma_{p}^{2}}{\sigma_{p}^{2}+\sigma_{e}^{2}}$, the correlation between individual observations. Observations are more characteristic of undesirable objects for lower values of $X_{0}$ : undesirable objects have an average phenotype of 0 , and desirable objects of 1 . The blue line indicates the attackflee threshold of a single step signal detection model. . . . . . . . . . . . 42 
3.2 The optimal action after having made a single observation of the prey $X_{0}=0.5$, at a background frequency of desirable prey of $p=0.7$ and with $\Delta=0.05$. The optimal action is either to attack immediately (green area of each panel), flee immediately (red area), or continue observing for $n^{*}$ additional observations (coloured by $n^{*}$, as in legend). Columns correspond to values of $\sigma(0)=\sqrt{\sigma_{p}^{2}+\sigma_{e}^{2}}$, the standard deviation of a single observation, and rows to $r=\frac{\sigma_{p}^{2}}{\sigma_{p}^{2}+\sigma_{e}^{2}}$, the correlation between individual observations. . . . . . . . . . . . . . . . . . 43

3.3 The optimal distance $d$ to make a single additional observation of the prey. Grey indicates $d=\infty$, i.e. fleeing immediately, and $d=0$ (dark purple) indicates attacking immediately. The background frequency of desirable prey is fixed at $p=0.7$ and the attack and escape probability coefficients at $\xi=0.05=\xi$. Columns correspond to values of $\sigma(0)=$ $\sqrt{\sigma_{p}^{2}+\sigma_{e}^{2}(1)}=\sqrt{\sigma_{p}^{2}+\epsilon^{2}}$, the standard deviation of the initial observation at $d=1$, and rows to $r=\frac{\sigma_{p}^{2}}{\sigma_{p}^{2}+\sigma_{e}^{2}(1)}=\frac{\sigma_{p}^{2}}{\sigma_{p}^{2}+\epsilon^{2}} . \ldots \ldots \ldots . \ldots . \ldots 46$

3.4 The optimal distance $d$ to make a single additional observation after having made an observation $X_{0}=0.5$ at a distance of 1 . Grey indicates $d=\infty$, i.e. fleeing immediately, and $d=0$ (dark purple) indicates attacking immediately. The background frequency of desirable prey is fixed at $p=0.7$ and the escape probability coefficient at $\delta=0.05$. Columns correspond to values of $\sigma(0)=\sqrt{\sigma_{p}^{2}+\sigma_{e}^{2}(1)}=\sqrt{\sigma_{p}^{2}+\epsilon^{2}}$, the standard deviation of the initial observation at $d=1$, and rows to $r=\frac{\sigma_{p}^{2}}{\sigma_{p}^{2}+\sigma_{e}^{2}(1)}=\frac{\sigma_{p}^{2}}{\sigma_{p}^{2}+\epsilon^{2}} .47$ 
B.1 Estimated palatabilities of a novel phenotype under a binary palatability model. $n$ and $m$ are the assumed number of populations of palatable and unpalatable prey respectively. The previous observations, palatable with phenotype -2 and unpalatable with phenotype 2 , are shown by the blue and red lines respectively. The phenotype is in units of $\sigma_{w} . \ldots \quad 74$

B.2 Estimated palatabilities of a novel phenotype under a binary palatability model. $n$ and $m$ are the assumed number of populations of palatable and unpalatable prey respectively. The previous observations, palatable with phenotype -0.5 and unpalatable with phenotype 0.5 , are shown by the blue and red lines respectively. The phenotype is in units of $\sigma_{w} . \quad \ldots 75$

D.1 The optimal action after having made a single observation of the prey. As Figure 3.2 , but with $\Xi=0.05$ and $\Delta$ on the y-axis. . . . . . . 80 D.2 The optimal distance $d$ to make a single additional observation after having made an observation at a distance of 1 . As Figure $\mid 3 \cdot 4$, but with

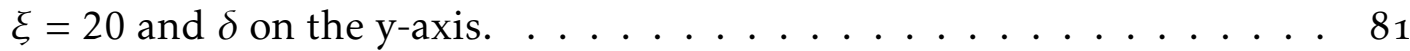

D.3 The optimal action after having made a single observation of the prey. As Figure 3.1 , but with $\Xi$ on the y-axis and $\left|v_{u}\right|=\left|v_{d}\right| . \ldots \ldots \ldots 2$

D.4 The optimal action after having made a single observation of the prey.

As Figure $\mid 3.1$, but with $\Delta$ on the $y$-axis and $\left|v_{u}\right|=\left|v_{d}\right| \cdot \ldots \ldots \ldots$

D.5 The optimal action after having made a single observation of the prey. As Figure 3.3 , but with $\xi$ on the y-axis and $\left|v_{u}\right|=\left|v_{d}\right| . \ldots \ldots \ldots$

D.6 The optimal action after having made a single observation of the prey. As Figure 3.1 , but with $\delta$ on the y-axis and $\left|v_{u}\right|=\left|v_{d}\right|$. $\ldots \ldots \ldots$

D.7 The optimal action after having made a single observation of the prey.

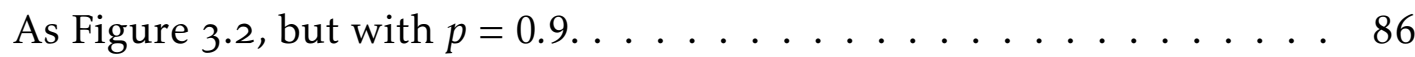


D.8 The optimal action after having made a single observation of the prey.

As Figure D.1, but with $p=0.9 . \ldots \ldots \ldots \ldots \ldots$ 


\section{INTRODUCTION: ANTI-PREDATOR DEFENCES AND PREDATOR DECISIONS}

Predator-prey interactions are ubiquitous in nature, and finding food while avoiding being eaten is a key component of the fitness of many organisms. Indeed, almost all organisms are potentially subject to predation, and avoiding predation is a significant challenge for them. This causes the development of anti-predator defences that prevent successful predation, either by avoiding interactions with predators or by preventing successful consumption (Ruxton et al. 2018). Conversely, predators depend upon their ability to consume sufficient prey for reproduction and growth, and must therefore find a way to overcome the defences of their prey. This produces a coevolutionary arms race between predators and prey (Brockhurst et al. 2014).

Predator-prey encounters are generally divided into several stages: encounter (when the predator comes within a distance where the prey is potentially detectable), detection of the prey, identification as suitable prey, attack, subjugation (catching the prey and preventing it from escaping), and consumption (Endler 1991). Defences can operate at any of these stages, and indeed many prey have "portfolios" of several defences operating at multiple stages (Ruxton et al. 2018). However, these defences come with costs to maintain (Bowers 1992; Cipollini et al. 2014; Blanchard and Moreau 2016). All defences have some degree of cost, and this is particularly true of secondary defences, those which take effect after the predator attacks, such as toxicity, defensive secretions, or spines and other mechanical 
defences. In addition to the cost to maintain these defences often being greater than that of primary defences which may be purely colouration or behavioural, defences that are late in the predation sequence may be more costly when they fail, as there is an increased risk of injury (Endler 1991; Broom et al. 2010). Even if the defence is successful there may be a cost. For example, toxicity as a defence only works if the predator at least tastes the prey: lucky prey might be taste rejected, but they might be consumed nevertheless (Holen 2013).

There is therefore an advantage to having defences that can be deployed early in the predation sequence, where direct interaction with the predator is not required. One example is crypsis, which prevents the predator from discovering the prey item, but there are also defences which act as signals of later defences in the sequence. The first of these is aposematism, in which unpalatability or unsuitability as prey is advertised by means of a conspicuous signal, such as bright colours (Poulton 1890; Edmunds 1974). Aposematic prey still incur some cost from training predators to associate their colouration (or other signal) with unpalatability. This cost can be reduced if multiple aposematic prey species converge on the same signal, a defence called Müllerian mimicry (Müller 1879; Aubier et al. 2017). Similarly, prey without a suitable secondary defence may still gain some advantages of aposematism by showing the aposematic phenotype, in what is called Batesian mimicry (Bates 1862).

Thus far, I have discussed the advantages of these anti-predator defences for prey. But there is also an advantage for predators to respond to them. Aposematic signals (including Müllerian mimics) are by definition honest signals of some form of unpalatability or defence, which the predator would be generally better to avoid, and are thus beneficial for both predator and prey. Not all defences, however, are mutually beneficial: Batesian mimics, of course, are taking advantage of 
predators' learned aversion by sending deceptive signals to predators to induce them to avoid perfectly good prey. In addition, predators may not always follow aposematic signals exactly: they may consume unpalatable prey when there are few alternative prey, or when the unpalatable prey are particularly nutritious (Barnett et al. 2007; Halpin et al. 2014). Predators thus may use aposematic signals to identify borderline suitable prey, contrary to their "intended" role (Guilford and Dawkins 1991; Skyrms and Barrett 2019). Conversely, these borderline suitable prey may gain protection by looking like fully defended prey species, in "quasiBatesian" mimicry intermediate between Batesian and Müllerian mimicry.

Predators are faced with an uncertain world in which they must do their best to grow and reproduce. The environment they inhabit generally contains suitable prey for them, but also dangerous, unpalatable, or unprofitable potential prey best left alone except in extremis. Some unsuitable prey are helpfully marked by aposematic signals, but many of these signals must be learned, and Batesian mimics also use them. Predators must unify the information they gather about potential prey, both directly and through sources like social learning (Skelhorn, Halpin, et al. 2016; Thorogood et al. 2017), and information about their own state, to make good decisions about which potential prey to attack and when to abandon those attacks. It is these decisions that will determine whether anti-predator defences are effective: however, most models of anti-predator defence focus on the prey and rely on simplistic assumptions about predator behaviour. In the subsequent chapters I will present models for the decision making of predators in two scenarios. The first deals with the problem of when to attack a potential prey item, based on its phenotype, in general, and the model developed will incorporate predator learning using a Bayesian framework. The Bayesian framework allows one to quantify the predator's knowledge and uncertainty about the prey present in the 
form of probabilities, to update the knowledge and the uncertainty on the basis of new information (indeed, the Bayesian methodology is sometimes described as the best way to learn from experience; Jaynes 2003), and to make precise probabilistic predictions based on its accumulated knowledge. The second deals with decisions in a specific situation, that of encountering a startle display, and will be modelled as an extension of signal detection theory (Green and Swets 1966). 


\section{TOO CLOSE FOR COMFORT: PREDATOR GENERALIZATION OF PREY PALATABILITY}

Predators are faced with a variety of potential prey which can vary greatly both in appearance and in their profitability as prey. Based on their experience, they must decide which potential prey are worth attacking and which are best avoided. Key to making that decision is generalizing from phenotypes of prey that have been sampled to predict the palatability of prey with novel phenotypes. The strategy used clearly must be optimized to avoid both over- and undergeneralization, although models of generalization involving optimal use of available information are rarely developed. In this chapter, I develop a Bayesian framework for generalization by predators, in which predators attempt to estimate the overall distribution of phenotypes and degrees of palatability in the population, and use this to make predictions about novel phenotypes. I also give examples of the application of the framework to modelling the phenomenon of peak shift in generalization and to reconstructing some empirical results on generalization.

To think is to ignore (or forget) differences, to generalize, to abstract.

Jorge Luis Borges, "Funes el memorioso"

\subsection{Introduction}

One of the simplest ways for a prey species to defend itself against predation is to not be worth eating - that is unprofitable, either because it is too difficult or en- 
ergetically costly to catch, or unpalatable or toxic when consumed (Ruxton et al. 2018). For predators, then, there will be a fitness benefit to being able to tell which prey are good to eat quickly and without having to sample them, so as to maximize their energy intake while minimizing the cost of attacking defended prey (Holen 2013). Thus, it is advantageous for predators to be able to learn from their experience with a small number of prey items to decide whether to pursue similar prey in the future. The key question is how similar? If they apply their knowledge of palatability only to absolutely identical prey items, then they will never use it (since even prey items of the same species may differ in appearance), whereas if they apply it to almost all prey, it provides no useful information; presumably the optimal degree of confidence in similarity of profitability will vary with similarity of appearance. This ability to apply learned responses to stimuli to similar novel stimuli is called generalization (Ghirlanda and Enquist 2003; Shepard 1987).

Generalization is particularly important to the ecology of predator-prey interactions because it allows for mimicry. When predators learn to avoid certain phenotypes of prey as unpalatable, it is advantageous for other prey to have similar phenotypes, whether they are undefended prey which will pass as defended (Batesian mimicry) or other defended prey which gain the advantage of faster predator learning or better memory by sharing similar appearances (Müllerian mimicry). These mimics need not even be all that similar in appearance: what is important is that predators do see them as similar enough to the model that they generalize their aversion to the latter into some degree of aversion to the former (Chittka and Osorio 2007). 


\subsubsection{Psychological aspects of generalization}

Generalization has been the subject of significant interest in psychology, largely as a purely mechanistic process, without considering optimality or fitness costs or benefits. Typically in animal experiments on generalization, novel stimuli of varying degrees of similarity to some fixed stimulus are presented to individuals, and their degree of generalization from the fixed stimulus is measured as the strength of response relative to that for the fixed stimulus. These fixed stimuli are generally of two types: either stimuli the subjects have prior experience with, or those they have been trained to respond to through differential training. In differential training, the subject is trained (by providing some reward) to perform some response to a stimulus $S^{+}$and is also presented during training with another stimulus $S^{-}$(often simply the absence of $S^{+}$), which is unrewarded. Experiments of this form have produced largely similar patterns of generalization across taxa, the sensory form of the stimuli, and the type of response and reward (Ghirlanda and Enquist 2003). This uniformity has produced great interest in creating models of generalization (Cheng 2002).

One of the most successful such models is that of Shepard (1987), which argues that the degree of generalization from one stimulus to another is a negative exponential function of the distance between the stimuli in an "internal psychological space" representing the individual's perception of the stimulus. He justifies this with both (i) an analysis of experimental data from several studies, in which nonmetric multidimensional scaling is used to obtain estimates of distances in psychological space from generalization data and shown to produce an exponential curve, and (ii) a theoretical model of generalization that produces roughly exponential generalization curves. Although there is some empirical evidence supporting the model (e.g. in honeybees; Cheng 2000), there is also evidence Gaussian 
generalization (where the degree of generalization is given by the negative exponential of the squared psychological distance) is more common than exponential generalization (Ghirlanda and Enquist 2003). Shepard (1987) proposes that these Gaussian cases are due to the combination of physical inability to discriminate between very similar stimuli with to true psychological generalization; others have proposed extensions to the model to account for different forms of generalization curve (e.g. Chatera and Vitányi 2003; Staddon and Reid 1990). In any case, the model is purely functional: it does not include fitness benefits or costs of any degree of generalization (and probably could not at the level of generality it includes), and therefore does not consider any evolutionary consequences of generalization. In short, it fails to show how generalization might arise as a consequence of the optimal use of available information.

One phenomenon observed in psychological studies of generalization is that of peak shift, also called supernormal stimulation (Staddon 1975). Peak shift occurs when a subject that has been differentially trained on a stimulus exhibits the greatest (peak) response not at $S^{+}$, but at a more extreme value of the stimulus; similarly, the greatest aversion or disinterest is not at $S^{-}$, but at a more extreme value (Spence 1937). The effect is consistently observed across taxa and types of stimuli (Ghirlanda and Enquist 2003; Baddeley et al. 2007; Cheng and Spetch 2002; Cheng et al. 1997; Spetch and Cheng 1998). The phenomenon requires differential training (i.e. the presence of an $S^{-}$) to occur, and the degree of shift (distance between $S^{+}$and the peak response) increases with decreased distance between $S^{+}$ and $S^{-}$(Cheng and Spetch 2002). This is typically explained by a model in which the subject not only generalizes from the $S^{+}$to assume that nearby stimuli are rewarded, but also from the $S^{-}$to assume nearby stimuli are unrewarded. The combination of these two generalization curves produces an overall curve that exhibits 
peak shift (Spence 1937). Leimar et al. (1986) proposed that peak shift is a contributory factor to the evolution of aposematism: a selective pressure for brighter and more conspicuous colouration is produced because predators will be even less likely to attack prey with even more extreme phenotypes after sampling unpalatable aposematic prey (there is some empirical evidence for this in e.g. Gamberale and Tullberg 1996; see also Mallet and Joron 1999). Yachi and Higashi (1998) incorporated this hypothesis into a mimicry model.

\subsubsection{Generalization and mimicry models}

Although generalization is of central importance to explaining the evolution of mimicry, it has been incorporated into models of mimicry relatively rarely. The earliest models of mimicry (e.g. Müller 1879; Marshall 1908) contained no aspect of generalization; phenotypes were assumed to be either perfect mimics or perfectly distinguished from each other, and each species had one fixed phenotype. Fisher (1927) invoked predator generalization to explain how Müllerian mimicry could evolve gradually; without generalization, mimicry must effectively be arrived at through phenotypic leaps directly to the phenotype of a defended species, which was the proposal of Punnett (1915). Two related hypotheses, gradual convergence of phenotypes and the "two-step" hypothesis of a large leap followed by fine tuning, introduced by Nicholson (1927), are still subject to debate today (Turner 1984; Balogh and Leimar 2005; Balogh et al. 2010; Leimar et al. 2012). A brief historical overview can be found in Ruxton et al. (2018, §9.4.10).

Even among models that include generalization, there has not been much interest in the source of generalization curves used in models. When generalization is included in a model, it is generally assumed that the predator generalizes its experience with a Gaussian curve of some fixed width centred on the consumed prey. 
Table 2.1: Comparison of generalization in models of mimicry.

\begin{tabular}{|c|c|}
\hline Generalization & Studies \\
\hline No generalization & 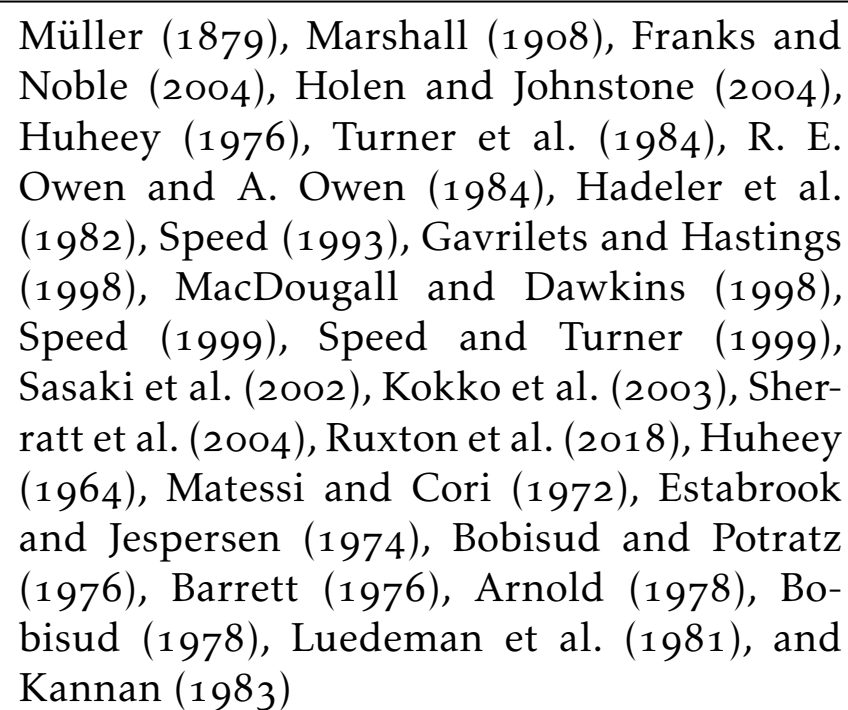 \\
\hline $\begin{array}{l}\text { Unspecified form of } \\
\text { generalization }\end{array}$ & $\begin{array}{l}\text { Fisher } 1927) \text { and Williamson and Nelson } \\
(1972)\end{array}$ \\
\hline Linear & Franks and Noble 2003 \\
\hline Gaussian & $\begin{array}{l}\text { Balogh and Leimar }(2005) \text {, Franks et al. } \\
(2009) \text {, Franks and Sherratt }(2007) \text {, Leimar et } \\
\text { al. (1986), and Yachi and Higashi }(1998)\end{array}$ \\
\hline Gaussian and Laplace & Ruxton et al. (2008) \\
\hline $\begin{array}{l}\text { Gaussian with feature } \\
\text { classification }\end{array}$ & $\begin{array}{l}\text { Balogh et al. (2010) and Gamberale-Stille et } \\
\text { al. (2012) }\end{array}$ \\
\hline
\end{tabular}

An extension proposed in Balogh et al. (2010) treats a certain region in phenotypic space as corresponding to a "feature," and predators generalize more narrowly for prey that both fall in the region (share the feature) than for those that don't. A list of mimicry models in the literature and aspects of predator generalization they include is found in Table 2.1. One exception to the general rule is Ruxton et al. (2008), in which the effects of Gaussian and Laplace generalization curves were compared (it was also shown that the prediction of Balogh and Leimar 2005) that Laplace generalization would not permit the gradual evolution of mimicry is false). 
In the remainder of this chapter, I will develop a model framework in which generalization is treated as a general problem of learning about the environment (Section 2.2). I then develop several example models within the framework: two models of peak shift (Section 2.3), and a reconstruction of some experimental results (Section 2.4).

\subsection{Model framework}

In this chapter, I develop a model framework that can be applied to problems of predator generalization. The general modelling strategy used is to build a model describing a predator's perception or estimation of the distribution of phenotypes among the prey in the environment. This is a natural way of looking at generalization: the predator has sampled some small number of prey, of which it knows the phenotypes and palatabilities; when generalizing from these samples it estimates the distribution of these traits in the entire population based on its experience. Of course, what the predator really needs to know to make decisions as to when to attack is the probability of prey of a given phenotype being unpalatable, which can be found from the estimated overall distribution by conditionalizing. This strategy lends itself to a Bayesian model based on the model of human inductive generalization developed by Sanjana and Tenenbaum (2003), which is further expanded in Tenenbaum and Griffiths (2001) and Kemp and Tenenbaum (2009).

\subsubsection{Background}

The model of Sanjana and Tenenbaum (2003) is as follows. Consider a finite set $S$ of possible stimuli, and a subset $C \subseteq S$ of those stimuli corresponding to a certain concept. For example, $S$ might be the set of all possible prey phenotypes, and $C$ those that correspond to unpalatable prey. The problem the model addresses is 
that of learning the concept $C$ from examples; that is, given a set $X=\left\{x_{1}, \ldots, x_{n}\right\} \subseteq C$ of $n$ stimuli that have been observed to be examples of the concept, to determine the probability that a new stimulus $y \in S$ is an example of the concept.

To do this, consider a hypothesis space $\mathcal{H}$, each element $h \in \mathcal{H}$ of which is a possible candidate for $C$, that is, a subset of $S$ that might correspond to the concept. For example, $h$ could be the set of all red phenotypes, corresponding to the hypothesis that the unpalatable prey are exactly the red prey. Then

$$
\begin{aligned}
P(y \in C \mid X) & =\sum_{h \in \mathcal{H}} P(y \in C \mid h) P(h \mid X) \\
& =\sum_{h \in \mathcal{H}: y \in h} P(h \mid X),
\end{aligned}
$$

where the first equality follows from the fact that the hypotheses are mutually exclusive (only one can be $C$ ), and the second follows because $P(y \in C \mid h)$ is just 1 if $y \in h$ and 0 otherwise. Then by Bayes' Theorem,

$$
P(y \in C \mid X)=\sum_{h \in \mathcal{H}: y \in h} \frac{P(X \mid h) P(h)}{P(X)} .
$$

To find $P(X \mid h)$, assume the $x_{i}$ are selected independently and uniformly from the possible stimuli. If any $x_{i}$ is not in $h$, then $h$ cannot be the correct candidate. If $h=C$, the probability of getting any particular $x_{i} \in h$ when selecting uniformly from $h$ is $1 /|h|$. Then since the $x_{i}$ are independent,

$$
P(X \mid h)= \begin{cases}0 & \text { if any } x \in X \text { is not in } h \\ (1 /|h|)^{n} & \text { otherwise. }\end{cases}
$$

Tenenbaum and Griffiths (2001) extends this in the natural way to the case where $S$ is not finite, but a bounded subset of $\mathbb{R}^{m} ; P(X \mid h)$ is then given by the uniform distribution on the set $h$.

Thus the results of the model depend heavily on two selections: 
- the choice of hypothesis space $\mathcal{H}$, and

- the choice of prior on the hypotheses, $P(h)$.

Sanjana and Tenenbaum (2003) give examples of these that are fairly specific to their example of inference for taxonomic categories, and Kemp and Tenenbaum (2009) discusses the general problem of deriving them.

\subsubsection{The extended model}

The model presented in the previous subsection is limited in two major ways: the set of stimuli must be a compact subset of $\mathbb{R}^{m}$, and the concepts learned are all simply a uniform distribution on some subset of stimuli. In this subsection, I extend the model by allowing for arbitrary stimuli and generalizing the hypotheses to arbitrary distributions. The latter has the additional advantage of allowing for the possibility that some phenotypes are expressed by both palatable and unpalatable prey. Suppose predators observe prey phenotypes from a space $\Phi$ (for now, this will always simply be $\mathbb{R}$ ). I also assume that each prey item has some fitness cost or benefit associated with consuming it, which the predator can observe by attacking the prey item. Thus each potential prey has a phenotype and a palatability, and corresponds to a point $x \in \Phi \times \mathbb{R}$. As before, the predator will observe a series of phenotypes of prey $x_{1}, \ldots, x_{n}$ on the basis of which it will estimate the joint distribution of phenotypes and palatabilities over the entire population.

As before, the hypothesis space $\mathcal{H}$ consists of candidates for the estimated quantity, so $\mathcal{H}$ consists of densities on $\Phi \times \mathbb{R}$. One also needs a prior distribution $\pi$ on $\mathcal{H}$ for the hypotheses giving the predator's initial guess at how likely each of the hypotheses is. If the predator observes (consumes) $n$ independent prey $x_{1}, \ldots, x_{n}$, 
its updated posterior distribution on $\mathcal{H}$ is given by

$$
\begin{aligned}
f\left(h \mid x_{1}, \ldots, x_{n}\right) & =\frac{f\left(x_{1}, \ldots, x_{n} \mid h\right) \pi(h)}{\int_{\mathcal{H}} f\left(x_{1}, \ldots, x_{n} \mid h\right) \pi(h) d h} \\
& =\frac{\pi(h) \prod_{i=1}^{n} h\left(x_{i}\right)}{\int_{\mathcal{H}} \pi(h) \prod_{i=1}^{n} h\left(x_{i}\right) d h},
\end{aligned}
$$

where the second equality is given by the fact that the $x_{i}$ are independently and identically distributed according to the true distribution of prey.

Suppose now that the predator has a distribution $f$ on $\mathcal{H}$ (either the prior $\pi$ or a posterior distribution after a certain number of observations), and it observes a prey of phenotype $\phi$. The expected fitness benefit $w$ from consuming the prey is then

$$
\mathbb{E}[w \mid \phi]=\int_{\mathcal{H}} \int_{\mathbb{R}} w h(w \mid \phi) f(h) d w d h,
$$

where for all $h$,

$$
h(w \mid \phi)=\frac{h(w, \phi)}{\int_{\Phi} h(w, \phi) d \phi} .
$$

Note that this expected value involves integrating over $\Phi \times \mathbb{R}$ with respect to $h$, which corresponds to actual environmental uncertainty in the palatability of the prey item assuming $h$ is the true distribution of prey, and over $\mathcal{H}$ with respect to $f$, which corresponds to subjective or epistemic uncertainty with respect to which distribution is the true one.

\subsubsection{Choosing priors}

The heart of the model lies in choosing the hypothesis space and its prior distribution. There are two special cases that will be used quite frequently in the remainder of the chapter. The first occurs when $\mathcal{H}$ consists of a parametric family of distributions, which I will call a parametric hypothesis space. Each element of $\mathcal{H}$ then is of the form $f(y \mid \theta)$, for a parameter $\theta$, and the prior distribution on $\mathcal{H}$ is then simply a prior on the parameter, $\pi(\theta)$. 
The second is the case where there are only two palatabilities: palatable prey give a fitness benefit when consumed, and unpalatable prey impose a fitness cost. Note that since there are only two possible values of palatability in this case, their actual values are irrelevant as long as the benefit is greater than the cost, because a change in one of the payoffs is equivalent to simply choosing different units for the palatability. In this case, which I shall call a binary palatability model, the distributions in $\mathcal{H}$ all have three components: a distribution $\epsilon$ on $\Phi$ giving the phenotypic distribution among palatable prey, a distribution $\delta$ on $\Phi$ giving the phenotypic distribution among unpalatable prey, and $p$, the proportion of prey that are palatable.

\subsection{Peak shift}

Since it is a commonly investigated and observed characteristic of generalization, I will explore peak shift as an example of modelling of generalization using the model framework just developed. I assume that the potential prey items can be subdivided into populations or types (because, for example, the prey community is made up of multiple species or morphs). The distributions of phenotypes and palatabilities within the populations will be given by a family of parametric distributions $f(y \mid \theta)$ on the space of phenotypes, which in this case will always be $\mathbb{R}$. One can then put a prior $\pi(\theta)$ on the space of parameters $\Theta$. Two other important distributions are defined in terms of $f$ and $\pi$ : the marginal distribution of the phenotype

$$
m(y)=\int_{\Theta} f(y \mid \theta) \pi(\theta) d \theta
$$


and the posterior distribution of the parameter after having made an observation $y$ from the population,

$$
p(\theta \mid y)=\frac{f(y \mid \theta) \pi(\theta)}{m(y)} .
$$

The distribution over the entire prey community is then a mixture of distributions $f\left(y \mid \theta_{i}\right)$

$$
\frac{1}{n} \sum_{i=1}^{n} f\left(y \mid \theta_{i}\right),
$$

for some $n$, the number of populations or types. Although it might seem a natural extension to allow $n$ to vary and give it its own prior, or to have arbitrary mixture weights, and not just $1 / n$, it turns out that both of these extensions can be left out without significant loss of generality; see the discussion in Appendix A.

The prior distribution on the compound parameter of the overall distribution is

$$
\prod_{i=1}^{n} \pi\left(\theta_{i}\right)
$$

on the parameter space $\Theta^{n}$. The joint distribution of the parameter and observation is just the product of these two

$$
\frac{1}{n} \sum_{i=1}^{n}\left[f\left(y \mid \theta_{i}\right) \prod_{j=1}^{n} \pi\left(\theta_{j}\right)\right] .
$$

Integrating out the parameter shows that the marginal distribution of the mixture is the same as that of a single component, because all of the components of the mixture are interchangeable. If the predator makes a single observation $y$, the posterior distribution of $\theta_{1}, \ldots, \theta_{n}$ is then

$$
\frac{1}{n} \sum_{i=1}^{n} \frac{f\left(y \mid \theta_{i}\right) \prod_{j=1}^{n} \pi\left(\theta_{j}\right)}{m(y)}=\frac{1}{n} \sum_{i=1}^{n}\left[p\left(\theta_{i} \mid y\right) \prod_{\substack{j=1 \\ j \neq i}}^{n} \pi\left(\theta_{j}\right)\right],
$$

the average over all populations of the posterior assuming that $y$ comes from population $i$. The final distribution needed is the posterior predictive distribution: 
the distribution of the next observation, $y^{\prime}$, conditional on the observation already made, $y$ :

$$
\begin{aligned}
\int_{\Theta^{n}}\left(\frac{1}{n} \sum_{i=1}^{n} f\left(y^{\prime} \mid \theta_{i}\right)\right) \frac{1}{n} \sum_{i=1}^{n}\left(p\left(\theta_{i} \mid y\right) \prod_{\substack{j=1 \\
j \neq i}}^{n} \pi\left(\theta_{j}\right) \int_{\frac{1}{n}} \int_{\Theta} f\left(y^{\prime} \mid \theta\right) p(\theta \mid y) d \theta+\frac{n-1}{n} m\left(y^{\prime}\right) .\right.
\end{aligned}
$$

This is, of course, a weighted mixture of the posterior predictive and marginal distributions of the individual components. The justification for this is that the observation $y$ the predator learned from came from one of the populations (mixture components; we don't know which one, nor does it matter, since they are interchangeable); then to predict a subsequent observation $y^{\prime}$, we update one of the mixture components, and leave the rest the same.

\subsubsection{A binary palatability model}

I now apply the facts about mixture distributions just developed to model peak shift. The model will be a binary palatability model, i.e. there will be two classes of prey with distinct palatabilities. Within each of these two classes, the phenotypic distribution will be a mixture. The individual mixture components will be normal distributions, with variance $\sigma_{w}^{2}$ (the within-population variance), and parameterized by an unknown mean. The prior distribution on the means will also be normal (selected for convenience, since it is the conjugate prior of the normal), with mean 0 and variance $\sigma_{p}^{2}$ (the prior variance). Since the units of phenotype are arbitrary, the values of $\sigma_{w}^{2}$ and $\sigma_{p}^{2}$ are not important on their own: one can take $\sigma_{w}^{2}$ as the unit of phenotype, and then the ratio $\sigma_{p}^{2} / \sigma_{w}^{2}$ is relevant. By conjugacy, the posterior distribution of the mean for a single population is $N\left(\frac{\sigma_{p}^{2}}{\sigma_{p}^{2}+\sigma_{w}^{2}} y, \frac{\sigma_{p}^{2} \sigma_{w}^{2}}{\sigma_{p}^{2}+\sigma_{w}^{2}}\right)$, and if $f\left(y \mid \mu, \sigma^{2}\right)$ is the density of the normal distribution, then the posterior predictive 
distribution of the entire mixture is

$$
\begin{aligned}
\frac{1}{n} \int_{\mathbb{R}} f\left(y^{\prime} \mid \mu, \sigma_{w}^{2}\right) f\left(\mu \mid \frac{\sigma_{p}^{2}}{\sigma_{p}^{2}+\sigma_{w}^{2}} y, \frac{\sigma_{p}^{2} \sigma_{w}^{2}}{\sigma_{p}^{2}+\sigma_{w}^{2}}\right) d \mu+\frac{n-1}{n} f\left(y^{\prime} \mid 0, \sigma_{w}^{2}+\sigma_{p}^{2}\right)= \\
\frac{1}{n} f\left(y^{\prime} \mid \frac{\sigma_{p}^{2}}{\sigma_{p}^{2}+\sigma_{w}^{2}} y, \frac{\sigma_{w}^{2}\left(2 \sigma_{p}^{2}+\sigma_{w}^{2}\right)}{\sigma_{p}^{2}+\sigma_{w}^{2}}\right)+\frac{n-1}{n} f\left(y^{\prime} \mid 0, \sigma_{w}^{2}+\sigma_{p}^{2}\right) .
\end{aligned}
$$

I will call this posterior predictive distribution $\epsilon\left(y^{\prime} \mid y\right)$ for the palatable prey. The distribution $\delta\left(y^{\prime} \mid y\right)$ for unpalatable prey will be of identical form, except that the number of mixture components may be different: I will denote the number of components $n$ for $\epsilon$ (i.e. $n$ is the number of populations of palatable prey) and $m$ for $\delta$ ( $m$ is the number of populations of unpalatable prey). Given the two posterior predictive distributions and the prior probability of a prey item being palatable, one can calculate the expected palatability of a new prey item with a given phenotype.

The results of this model for a simple situation are shown in Figure 2.1 the exact numerical locations of the peaks are shown in Table 2.2. The predator has observed one palatable prey with phenotype -1 , and one unpalatable with phenotype 1 , and now will estimate the palatability of a new prey item with any phenotype. The most important aspect is immediately visible: in the cases with only one unpalatable mixture component, the degree of peak shift at the palatable peak is infinite, and vice versa. Once multiple mixture components are present, there is a peak at a finite phenotype value where the extremum is attained. The overall pattern is that adding more palatable populations increases the degree of peak shift of the palatable peak, while adding more unpalatable populations decreases it (the complementary statements are true of the unpalatable peak). In addition, flatter priors (larger prior variance) produce higher peaks and the degree of peak shift varies non-linearly with the prior variance. The model also produces the classical peak shift result that stimuli closer together produce greater degrees of shift (see the supplementary figures B.1 and B.2 and table B.1. 


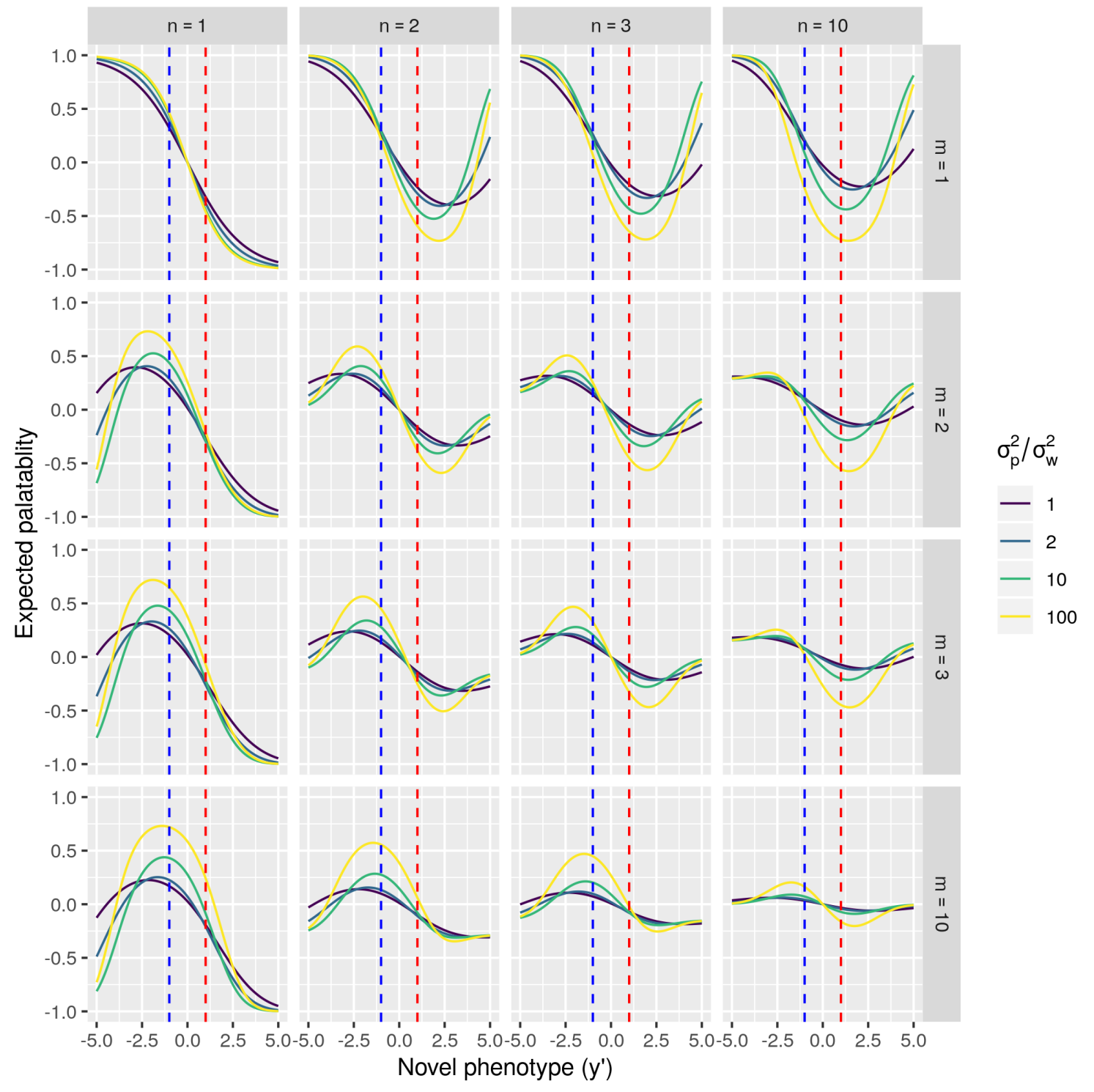

Figure 2.1: Estimated palatabilities of a novel phenotype under a binary palatability model. $n$ and $m$ are the assumed number of populations of palatable and unpalatable prey respectively. The previous observations, palatable with phenotype -1 and unpalatable with phenotype 1 , are shown by the blue and red lines respectively. The phenotype is in units of $\sigma_{w}$. 
Table 2.2: Degrees of peak shift from a binary palatability model. The peak locations and values are given for the palatable peak (the unpalatable peaks can be extrapolated by symmetry). $n$ and $m$ are the assumed number of populations for palatable and unpalatable prey respectively. The peak shift is the distance between the peak location and the training example phenotype, which was always -1 .

\begin{tabular}{|c|c|c|c|c|c|}
\hline$n$ & $m$ & $\sigma_{p}^{2} / \sigma_{w}^{2}$ & Peak location & Peak shift & Peak value \\
\hline \multirow[t]{9}{*}{2} & \multirow[t]{3}{*}{2} & 1 & -3.22 & 2.22 & 0.334 \\
\hline & & 10 & -2.12 & 1.11 & 0.407 \\
\hline & & 100 & -2.31 & 1.31 & 0.590 \\
\hline & \multirow[t]{3}{*}{3} & 1 & -2.80 & 1.80 & 0.239 \\
\hline & & 10 & -1.81 & 0.805 & 0.340 \\
\hline & & 100 & -2.00 & 0.997 & 0.564 \\
\hline & \multirow[t]{3}{*}{10} & 1 & -2.25 & 1.25 & 0.140 \\
\hline & & 10 & -1.34 & 0.344 & 0.285 \\
\hline & & 100 & -1.44 & 0.441 & 0.573 \\
\hline \multirow[t]{9}{*}{3} & \multirow[t]{3}{*}{2} & 1 & -3.52 & 2.52 & 0.316 \\
\hline & & 10 & -2.31 & 1.31 & 0.360 \\
\hline & & 100 & -2.42 & 1.42 & 0.507 \\
\hline & \multirow[t]{3}{*}{3} & 1 & -3.03 & 2.03 & 0.213 \\
\hline & & 10 & -1.95 & 0.947 & 0.280 \\
\hline & & 100 & -2.08 & 1.08 & 0.468 \\
\hline & \multirow[t]{3}{*}{10} & 1 & -2.34 & 1.34 & 0.108 \\
\hline & & 10 & -1.40 & 0.404 & 0.213 \\
\hline & & 100 & -1.48 & 0.484 & 0.470 \\
\hline \multirow[t]{9}{*}{10} & \multirow[t]{3}{*}{2} & 1 & -4.73 & 3.73 & 0.309 \\
\hline & & 10 & -3.14 & 2.14 & 0.312 \\
\hline & & 100 & -3.04 & 2.04 & 0.346 \\
\hline & \multirow[t]{3}{*}{3} & 1 & -4.01 & 3.01 & 0.186 \\
\hline & & 10 & -2.62 & 1.62 & 0.195 \\
\hline & & 100 & -2.57 & 1.57 & 0.255 \\
\hline & \multirow{3}{*}{10} & 1 & -2.83 & 1.83 & 0.061 \\
\hline & & 10 & -1.75 & 0.746 & 0.090 \\
\hline & & 100 & -1.74 & 0.744 & 0.204 \\
\hline
\end{tabular}




\subsubsection{A second model}

An alternative way to apply the mixture model is to divide the prey community into populations that each have a distinct distribution of phenotypes and palatabilities, as opposed to the previous subsection's method of dividing them into palatable and unpalatable first and then into populations. A joint distribution of phenotypes and palatabilities is a distribution on $\mathbb{R}^{2}$. In this model, I will select the population distributions to be bivariate normal distributions with unknown means and covariance matrix

$$
\Sigma_{w}=\left[\begin{array}{cc}
\sigma_{w \phi}^{2} & 0 \\
0 & \sigma_{w w}^{2}
\end{array}\right] .
$$

The prior on the means will be the conjugate prior for the mean of a multivariate normal distribution, $\mathcal{N}\left(0, \Sigma_{p}\right)$, where the prior covariance matrix is

$$
\Sigma_{p}=\left[\begin{array}{cc}
\sigma_{p \phi}^{2} & 0 \\
0 & \sigma_{p w}^{2}
\end{array}\right] .
$$

Again, since the units of both palatability and phenotype are arbitrary, only the ratios of the corresponding variances matter. Then $\mathcal{H}$ will be the space of $n$ component mixtures of these distributions. After making an observation $y=(\phi, w)$, the posterior distribution of a single component is

$$
\mathcal{N}\left(\left(\Sigma_{p}^{-1}+\Sigma_{w}^{-1}\right)^{-1} \Sigma_{w}^{-1} y,\left(\Sigma_{p}^{-1}+\Sigma_{w}^{-1}\right)^{-1}\right)
$$

The posterior predictive distribution of the overall mixture can then be found as described at the beginning of the section. In this model, the posterior predictive distribution is a distribution of $y^{\prime}$, that is a joint distribution of phenotype and palatability. If the predator observes a particular $\phi^{\prime}$, it can then conditionalize to predict the palatability $w^{\prime}$. 


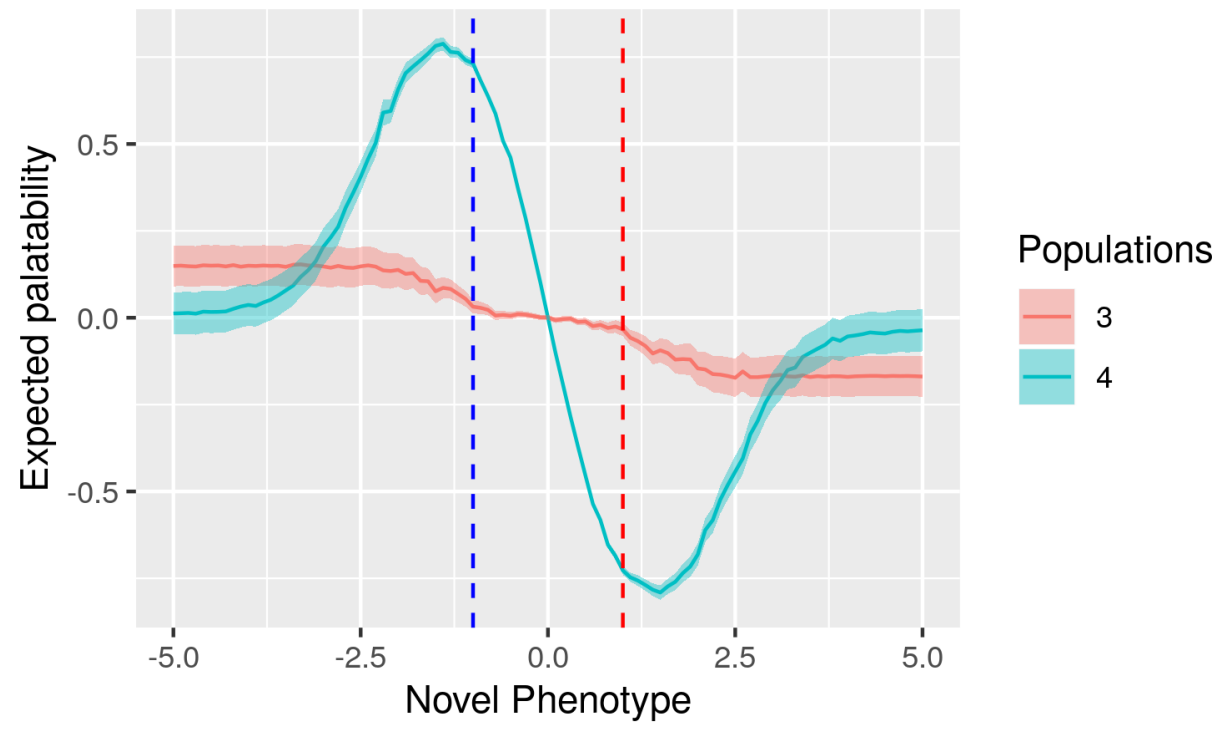

Figure 2.2: Expected palatability for varying phenotypes. Coloured bands are the $95 \%$ confidence intervals of the mean (solid line). The two solid lines correspond to models with mixtures of three and four normal distributions, respectively, and the two dashed lines to the locations of the previously observed palatable (blue) and unpalatable (red) prey.

I will look at a specific case of the model, with $n=3$ or $n=4$ components in the mixture, and $\sigma_{p \phi}^{2} / \sigma_{w \phi}^{2}=\sigma_{p w}^{2} / \sigma_{w w}^{2}=10$. Assume the predator has already made ten observations, five each at $(\phi, w)=(-1,1)$ and $(\phi, w)=(1,-1)$. This model is analytically intractable, since (i) the integral involved in finding $\mathbb{E}\left[w^{\prime} \mid \phi^{\prime}\right]$ is intractable, and (ii) learning from more than one sample makes the mixture model no longer tractable. The expected palatability was therefore estimated using Hamiltonian Monte-Carlo with the Stan software package (Carpenter et al. 2017).

As can be seen from Figure 2.2, at least 4 populations are required to observe a peak shift effect in this model. In the previous model, to see a peak required a palatable population, an unpalatable population, and at least one other population. In this model, it requires two additional populations. A probable explanation of this observation is that a single additional population has its effect averaged out, 
as it can lie beyond the observed stimuli in only one of the directions, whereas if there are two additional populations, each can lie beyond in a different direction.

\subsection{A reconstruction of empirical results}

In this section, the generalization model framework will be applied to experimental results from a study of colour generalization in chicks (Baddeley et al.2007). In this case, the phenotype will be colour, which will be considered as the response of the three types of single cone cells (short-, medium-, and long-wavelengthsensitive) in the chick's eyes. Since only the colour and not the brightness of the stimulus was taken into account, the phenotype space is a simplex (Figure 2.3 A). I examine the experiments 1 and 2 of Baddeley et al. 2007.

In both experiments, the chicks were first trained by being exposed to paper containers in the rewarded and unrewarded colours; the containers in rewarded colours contained food rewards, and those in unrewarded colours didn't. Training consisted of 24 exposures to each of the rewarded an unrewarded colour. The chicks were then tested with empty paper containers in four different colours. In experiment 1 , the chicks were divided into four groups, corresponding to the four labelled colours in Figure 2.3 B. All groups had the colour marked + as a rewarded colour. Chicks in group $n$ had colour $n$ as the unrewarded colour. In groups 1 and 2 , the test colours were $2,+, 3$, and 4 ; in groups 3 and 4 they were $3,+, 2$, and 1 (thus the test colours in group $n$ were + , the two colours on the opposite side of + from $n$, and either $n$ or the colour adjacent to it, whichever was closer to + ). In experiment 2, the chicks were divided into two groups, corresponding to the two - in Figure $2.3 \mathrm{C}$. The rewarded colour was + , the unrewarded colour in group $n$ was that marked $n$, and the novel colours were $2,+, \mathrm{N}_{1}$, and $\mathrm{N}_{2}$.

The model used here is both a parametric hypothesis space model and a binary 


\section{B: Experiment 1}

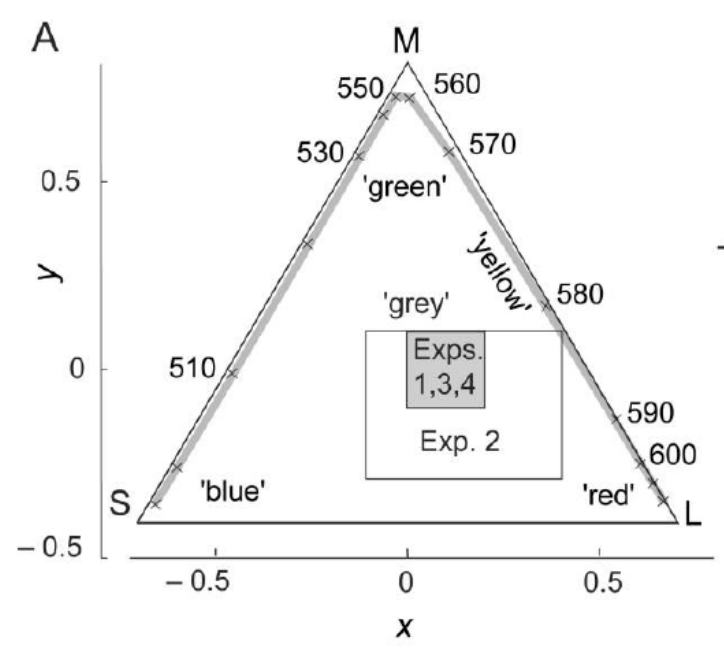

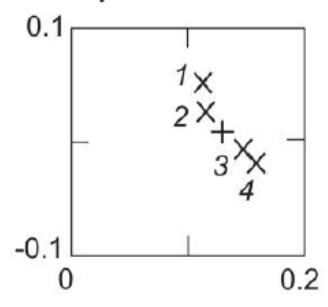

D: Experiment 3

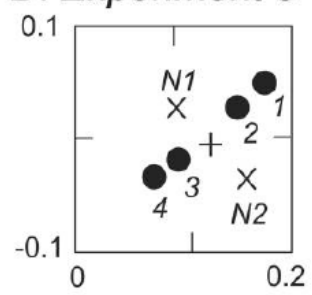

C: Experiment 2

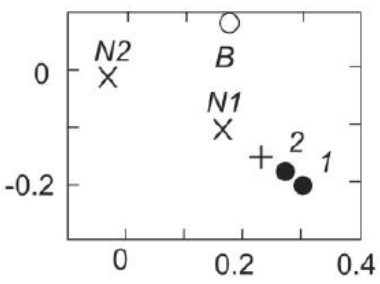

\section{E: Experiment 4}

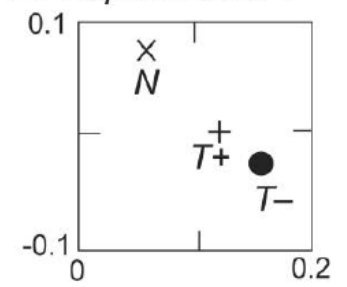

Figure 2.3: The colour experiment of Baddeley et al. (2007); reproduced from Baddeley et al. (2007), Figure 2. Panel A shows the colour space simplex; the grey line indicates the locations of monochromatic light at the indicated wavelengths $(\mathrm{nm})$. Panels B and C show the colours used in experiments 1 and 2. The rewarded colour in training is indicated with a + in both panels. In panel $\mathrm{B}$, the numbered $x$ show the respective unrewarded/test colours. In panel $C$, the unrewarded training colours are shown as $\bullet$, and novel colours as $\times$.

palatability model. It is assumed that there are two types of stimuli, rewarded and unrewarded (palatable and unpalatable). Within each type there is a distribution of phenotypes, which is a distribution on the two-dimensional simplex $\Delta$. Two models will be considered: in the first the phenotypic distribution within each type is a Dirichlet distribution, and in the second it is a mixture of two Dirichlet distributions. The Dirichlet distribution on $\Delta$ is parametrized by $\alpha \in\left(\mathbb{R}^{+}\right)^{3}$. This can be factored as $\alpha=a \hat{\alpha}$, with $\hat{\alpha} \in \Delta$ the mean of the Dirichlet and $a \in \mathbb{R}^{+}$a measure of concentration. We place a uniform prior on $\hat{\alpha}$ and a $\Gamma(4,0.02)$ prior on $a$ (the latter is a weakly informative prior suggesting that the concentration is very high, i.e. the populations of prey are uniform in colour, because the various stimuli are quite close together in the colour space). Then the model can be "trained" 
with 24 rewarded and 24 unrewarded stimuli of the appropriate colours (just as the chicks experienced), and the posterior expected palatability of a test colour measured.

The model was fitted to the experimental set-up using Hamiltonian MonteCarlo with Stan (Carpenter et al. 2017). The results are shown, compared to the experimental results from Baddeley et al. (2007), in Figures 2.4 and 2.5. In experiment 1 , the model with a mixture of two Dirichlet distributions qualitatively approximates the experimental results better, while in experiment 2 , the single Dirichlet model is the better approximation. In both cases, the model shows a more obvious peak shift than the experimental results.

\subsection{Discussion}

Generalization is an important adaptation to living in an uncertain world, where one must attempt to make accurate decisions based on limited experience. For predators, generalization is vital to determining as safely as possible which items in the environment are suitable prey. This in turn means that opportunities to take advantage of generalization, for example by mimicry, are key adaptations for prey. The adaptive function of generalization needs to be taken into account in models of it, and generalization needs to be taken into account when modelling anti-predator defence.

In this chapter, I have introduced a general framework for Bayesian modelling of generalization. The advantage of the Bayesian framework is twofold: first, it allows a decision theoretic approach, where decisions on whether to attack prey are based on the expected fitness outcomes, and second, it allows for a principled representation of predator's knowldege, uncertainty, and learning: instead of simply fixing a Gaussian or Laplace curve that defines some vague idea of similarity be- 

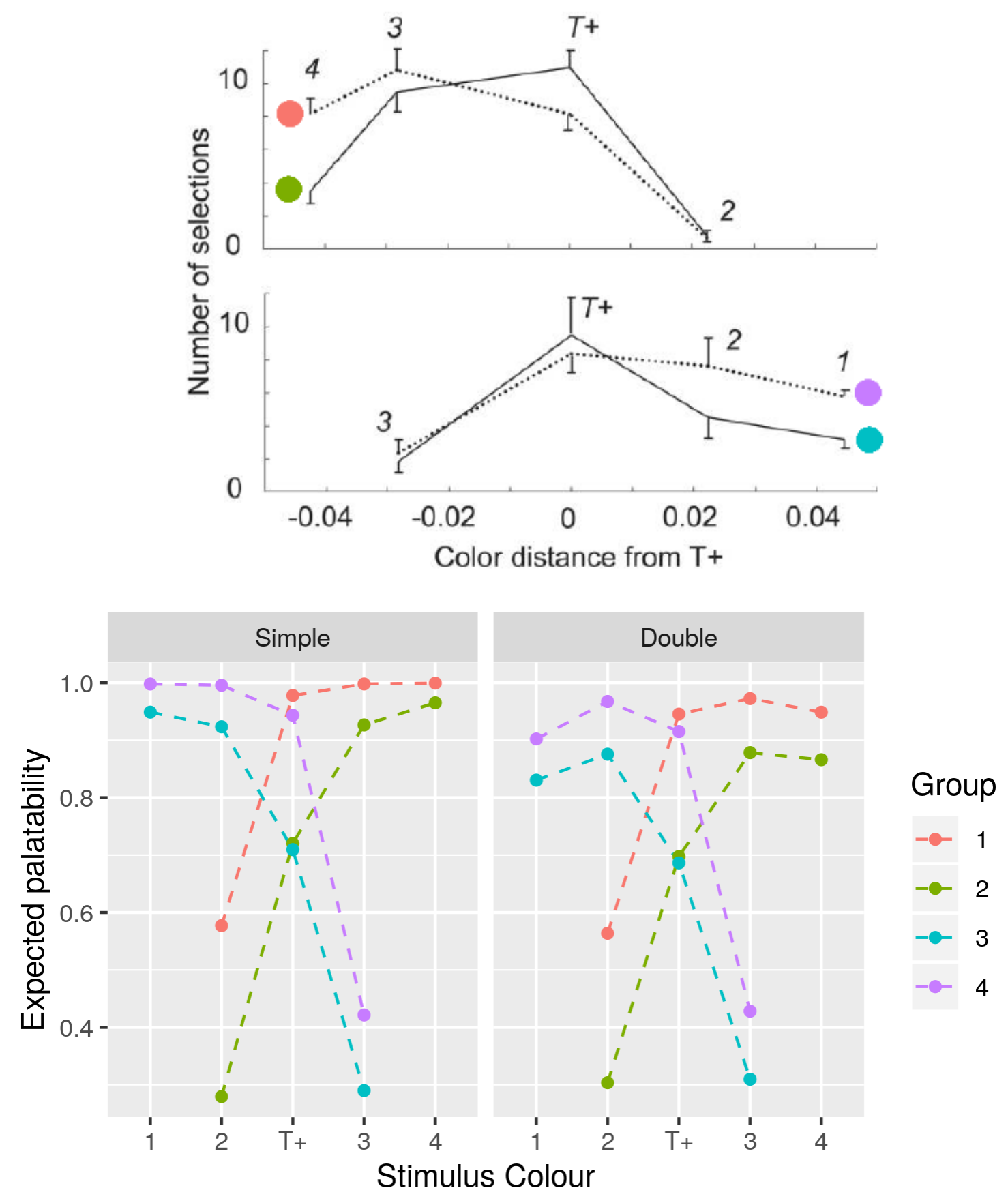

Figure 2.4: Results, real and simulated, of experiment 1 . The upper panel (from Baddeley et al. (2007), Figure 3a) shows experimental generalization in groups 1 (top dotted), 2 (top solid), 3 (bottom solid), and 4 (bottom dotted). The lower panel shows simulated generalization, in single Dirichlet per type (Simple) and two Dirichlet per type (Double) versions. 

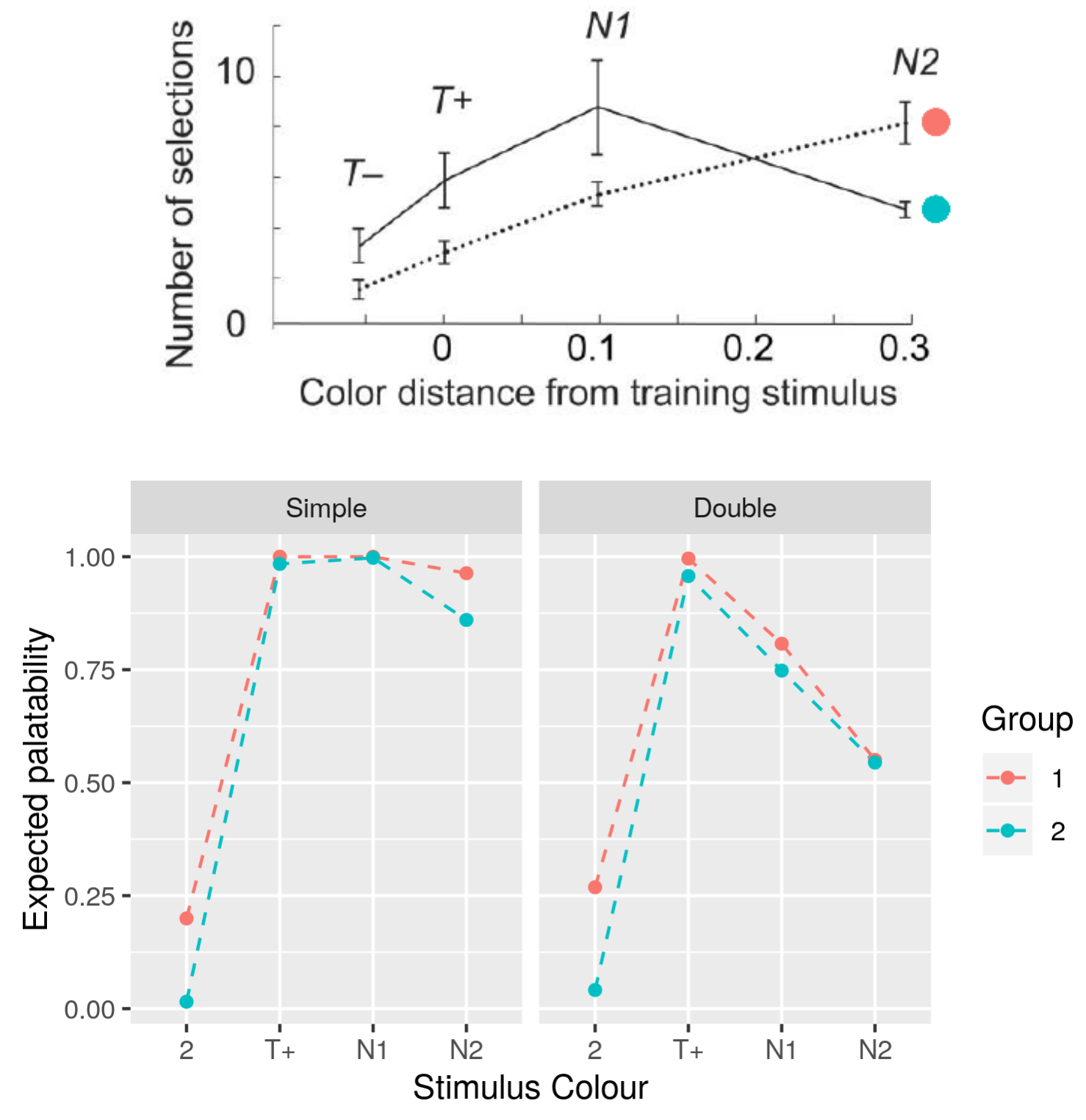

Figure 2.5: Results, real and simulated, of experiment 2. The upper panel (from Baddeley et al. (2007), Figure 4) shows experimental generalization in groups 1 (dotted), and 2 (solid). The lower panel shows simulated generalization, in single Dirichlet per type (Simple) and two Dirichlet per type (Double) versions. 
tween phenotypes, the predator's state of knowledge about the prey population can be represented as probabilities, and updated based on its experience and the solid foundation of Bayesian inference (Jaynes 2003). While some previous models have been developed in a Bayesian style, they fail to incorporate these components or include them in a very limited fashion. Sanjana and Tenenbaum (2003) do not include any adaptive component in their model, and although they consider learning, their focus on human inductive generalization means that it is in a very limited context. Baddeley et al. (2007) do not consider either of these components. Their model is similar to a restricted subset of the model of Subsection 2.3.1, with two groups each of rewarded and unrewarded stimuli, although they distinguish one of the groups as the one that was observed in training, and place different priors on the two (including an improper uniform prior on the unobserved group). A later model by some of the same authors, Scholtyssek et al. (2016), is very similar in its novel yet somewhat limited use of Bayesian methods.

In addition, the model framework is extremely general, and specific models based on it should be applicable to a very wide variety of circumstances. Specific subsets of the model framework, such as those I have called binary palatability models, or the mixture models considered in Section 2.3, can be applied to suitable problems. One such phenomenon that can be investigated through generalization models is peak shift. In this chapter, I showed that adaptive (in the sense of optimal use of information) peak shift depends not only on the interaction of a distribution of palatable and a distribution of unpalatable stimuli, as in the classical description, but also on the palatable and unpalatable distributions being multi-modal: in order for there to be a peak, there must be the possibility that there is another population, with distinct palatability, further beyond the location of the peak in the phenotype space. Another possible application of the model framework would 
be to examining taste rejection, when an unpalatable prey item is rejected after it has already been attacked (Skelhorn and Rowe 2009; Halpin and Rowe 2010). In terms of the Bayesian model, taste is of course just another aspect of phenotype that can be included in exactly the same fashion as any other. But biologically, taste is a much more accurate source of information on palatability, and obtaining information about taste requires an investment intermediate between that of simply observing a potential prey item and that of attacking outright. The accuracy of taste as a source of information could be incorporated by adding a correlation between the phenotype and palatability in the prior (in the examples considered here, they are uncorrelated in the prior, and any correlation comes from the evidence gathered). To incorporate the cost of tasting would require an extension of the model to include sampling costs.

Although the model framework is extremely general, there are areas in which it could be extended. One of these is interpreting the results of the model. The information the model provides is an estimated palatability (or distribution of palatabilites) for every phenotype, based on the predator's experience. The predator's optimal response is then binary: if the expected palatability is greater than the baseline fitness cost or benefit of doing nothing, attack, otherwise do not. But this is not the kind of behaviour exhibited in real generalization tasks. As shown in Figures 2.4 and 2.5, generalization data take the form of numbers or rates of attacks, and the rates are not always (or even often) zero or one. In this chapter, I have simply assumed that the rate of attack is roughly proportional to the expected palatability (this is a standard assumption; see e.g. Dittrich et al. 1993; Scholtyssek et al. 2016; Baddeley et al. 2007). A more principled approach would be to combine the model with signal detection or optimal foraging theory (Edwards 1965; Castellano 2019: Holen 2013) or exploration-exploitation models (Sherratt 2011), 
to make predictions that are directly interpretable in terms of experimental outcomes. 


\section{THE ADAPTIVE ADVANTAGE OF STARTLING AND BEING STARTLED}

Startle (deimatic) signals are used by a variety of species as an anti-predator defence, causing predators to delay or abandon their attack. One possible reason for the effectiveness of these signals is that they indicate a potential imminent harm. Because the display could be a genuine sign of danger or a bluff, the would-be predator faces a tradeoff between making a decision (attack or flee) quickly (to avoid being harmed or to successfully catch the prey) and taking time to examine the prey more carefully. We develop an extension of classical signal detection theory to multi-step problems to model this trade-off. The optimal behaviour for predators is affected by the degree to which the ambiguity in the signal is due to perceptual error, which can be reduced by further examination, and inherent variability in the types of stimuli in the environment, which cannot. We elucidate the circumstances in which immediate abandonment is a superior strategy to further examining a potential prey item or attacking it outright, and discuss the implications of the model for the evolution of startle signals.

... fear has sharp eyes, and sees things underground, much more above in heavens.

Miguel de Cervantes, Don Quixote de la Mancha 


\subsection{Introduction}

Startle displays (also called deimatic displays, from the Greek $\delta \epsilon \iota \mu \alpha \tau o ́ \omega$, "I frighten"; Maldonado 1970 Liddell and Scott 1940) are a form of anti-predator defence in which prey produce a sudden, conspicuous signal upon being approached by a predator (usually some sort of radical change in phenotype), which causes the predator to delay or break off attacking (Ruxton et al. 2018; Umbers et al. 2017). The classic example are the eyespots of many species of Lepidoptera, which are often hidden at rest and displayed only when approached by a predator (Olofsson et al. 2012). Eyespots are also found in a variety of other taxa (Skelhorn, Holmes, Hossie, et al. 2016), and other forms of deimatic display are also found in insects (Maldonado 1970; Kang et al. 2016; Umbers and Mappes 2015), molluscs (Staudinger et al. 2011), amphibians, fishes, etc. (Edmunds 1974), including deimatic displays in non-visual sensory modalities (Dookie et al. 2017).

In order to be a startle display, a defensive signal must actually function by startling a predator (as opposed, for example, to being purely an aposematic signal), and demonstrating the existence of a startle display therefore requires considering the behavioural responses of predators (Skelhorn, Holmes, and Rowe 2016). This is typically established by showing that predators exhibit unambiguous startle behaviours such as a quick retreat or alarm calls when exposed to the signals (Olofsson et al. 2012; Kang et al. 2017), or that they show habituation when repeatedly exposed to the same signal (Dookie et al.2017).

Why do these startle displays continue to work? What advantage is there to a predator to being startled, particularly when susceptibility to being startled clearly carries the cost of losing potential prey? A plausible answer is that it is due to two trade-offs with which predators are faced. The first is a speed-accuracy 
trade-off, in which high accuracy of identification requires low speed (Ruxton et al. 2018; Chittka and Osorio 2007). Gathering enough information to determine the true nature of a prey item exhibiting a startle display requires time, which in turn has costs (Abbott and Sherratt 2013; Chittka et al.2009). Moreover, if the potential cost of being attacked by an ambush predator is high enough, it may be advantageous to err on the side of treating startle displays as potential threats immediately (Janzen et al. 2010). Even if the predator opts for accuracy over speed and the potential prey item is really desirable, the predator's delay while it examines a prey item that has made a startle display may give the prey time to escape. There has been disagreement about the extent to which a startle display resembling a particular predator, as opposed to merely being salient, is necessary to impose costs on decision time, particularly in the case of eyespots (De Bona et al. 2015: Hossie et al. 2013; Stevens et al. 2009; Stevens and Ruxton 2014). Either way, however, the underlying speed-accuracy trade-off produces an adaptive advantage of the startle mechanism in avoiding predation.

The other key trade-off faced by the predator is between the frequencies of the two errors it can make when it finally stops its examination and makes a decision as to whether to attack or flee: misidentifying suitable prey as potential dangers and vice versa. This trade-off can also contribute to the development of startle displays in substantially the same manner as the speed-accuracy trade-off. The classical model of optimal discrimination between classes of stimuli is signal detection theory (Green and Swets 1966), which has been used extensively in modelling problems in behavioural ecology (Getty 1985; Wiley 1994; Fawcett and Johnstone 2003; Holen and Johnstone 2004; McGuire et al. 2006; Abbott and Sherratt 2013). Some work has also been done on models of speed-accuracy trade-offs alone (Edwards 1965; Froment et al. 2014; Gendron and Staddon 1983; Palmer et 
al. 2005), and on combining speed-accuracy trade-offs with signal detection theory, including simple models in which samples have constant costs (Getty 1996), models which incorporate optimal foraging to maximize energy intake (Holen 2013; Getty 1985), and incorporating opportunity costs of continued examination (Abbott and Sherratt 2013). In addition to combining speed-accuracy trade-offs with signal detection theory, they have also been incorporated into other decision making model frameworks, such as sequential sampling in the "Gruffalo" model of Castellano and Cermelli (2015). Here, we develop (and solve exactly) a novel, simple extension of classical signal detection theory to the case where a speedaccuracy trade-off is present due to a threat from the object being examined and examine its implications for the evolution of startle displays.

\subsection{The model}

Suppose a predator encounters a potential prey item that has just engaged in a deimatic display. The potential prey will fall into one of two classes: either it is desirable, and will give a fitness benefit $v_{d}>0$ when attacked (e.g. because it is actually palatable prey item), or it is undesirable, and will impose a fitness cost $v_{u}<0$ when attacked (e.g. because it is in fact a disguised ambush predator or because it is distasteful). The proportion of potential prey that are desirable will be denoted by $p$. The predator will make its decision based on some observed property or phenotype of the potential prey item, which is a real number. A signal detection model is based on an assumption about the distributions of this phenotype in the two classes. In this case, we use a normal-normal signal detection model, in which the distributions are normal in both classes, with identical variances, but distinct means. We fix the mean to be 0 for undesirable objects and 1 for desirable objects (this involves no loss of generality, because the units of the phenotype are 
arbitrary).

In order to account for the possibility of gathering more information about potential prey by continued examination, the variance of these distributions is assumed to come from two sources: variation between the phenotype of different objects within the same class, and variation between observations of the same object. The first type of variation we will call phenotypic variation, as it corresponds to variability in the true phenotype within each population, and will have variance $\sigma_{p}^{2}$. The second we will call error variation, as it corresponds to variability due to perceptual error in examining a single object, and will have variance $\sigma_{e}^{2}$.

Let $D$ be an indicator variable which is 1 if the potential prey item the predator is examining is actually desirable, and 0 if it is actually undesirable. Then

$$
D \sim \operatorname{Bernoulli}(p)
$$

By a less than fortuitous coincidence, $D$ is also the mean phenotype for whichever class the potential prey actually belongs to, and therefore its true phenotype unaffected by any perceptual error, $T$, is distributed as

$$
T-D \sim N\left(0, \sigma_{p}^{2}\right)
$$

Instead of observing the true phenotype directly, however, the predator has some degree of perceptual error, so that it makes observations $X_{0}, X_{1}, \ldots$ distributed as

$$
X_{n}-T \sim_{i i d} N\left(0, \sigma_{e}^{2}\right) .
$$

We further assume that the offsets $T-D$ and $X_{n}-T$ are independent from each other and from $D$. This means that, overall, the distribution of a single observation of a potential prey item is a mixture of two normal distributions: one with mean 1 and mixture weight $p$, and one with mean 0 and mixture weight $1-p$, both with variance $\sigma_{p}^{2}+\sigma_{e}^{2}$. 
Finally, we add a cost of sampling to the model; this is an important component because if there were no cost to sampling, it would be optimal to sample for an arbitrarily long time, to decrease the possibility of an erroneous decision as much as possible. This cost comes from two sources. First, we assume that undesirable objects are also possibly dangerous: after each additional observation $X_{i}$ made, an actually undesirable object has a probability $\Xi$ of inflicting the cost $v_{u}$ on the predator, and ending the interaction, irrespective of whether the predator decides to attack (this corresponds to an event like an ambush predator attacking the predator; we assume that being attacked by and mistakenly attacking a dangerous potential prey item have about the same costs, so we can use $v_{u}$ for both, which simplifies the math). Second, we assume that desirable prey may escape if the predator delays: after each observation, an actually desirable object will escape and end the interaction with probability $\Delta$. Naturally, if observation of the potential prey item has continued for some time and it has not escaped or attacked, then this information provides the predator more evidence about the probability of the potential prey item being desirable or undesirable (see below for how this is incorporated).

\subsubsection{The expected reward}

The predator wishes to determine whether the potential prey item it is examining is a desirable prey item which it should attack — that is to make an inference about the value of $D-$ based on some number of observations $X_{0}, \ldots, X_{n}$. The mean of the observations $\bar{X}_{n}=\frac{1}{n+1} \sum_{i=0}^{n} X_{i}$ is a sufficient statistic for $D$, so this inference can be made based on the mean alone. Since $\bar{X}_{n}=T+\frac{1}{n+1} \sum_{i=0}^{n}\left(X_{i}-T\right)$,

$$
\bar{X}_{n}-D \sim N\left(0, \sigma_{p}^{2}+\frac{\sigma_{e}^{2}}{n+1}\right) .
$$


We call the variance of this value $\sigma^{2}(n)=\sigma_{p}^{2}+\sigma_{e}^{2} /(n+1)$. This variance decreases as $n$ increases, because the variability due to perceptual error is eliminated by repeated sampling, but is bounded below by $\sigma_{p}$, because the phenotypic variation cannot be so eliminated. This is exactly a normal-normal signal detection problem, where the signal (the mean of the $n+1$ observations) comes from either a desirable distribution with mean 1 and variance $\sigma^{2}(n)$ or an undesirable distribution with mean 0 and variance $\sigma^{2}(n)$. The optimal decision strategy is known exactly in this case, as a classical result of signal detection theory (Green and Swets 1966): the expected fitness reward of attack is positive if and only if the observed mean is greater than a certain threshold, namely

$$
\lambda_{n}=\frac{1}{2}-\sigma^{2}(n) \log \frac{\pi_{n}\left|v_{d}\right|}{\left(1-\pi_{n}\right)\left|v_{u}\right|} .
$$

Here, $\pi_{n}$ is the prior probability that the potential prey item is desirable before considering the evidence of the current set of observations. Obviously, $\pi_{0}=p$. For $n \geq 1, \pi_{n}$ includes an additional source of information, namely the fact that the interaction has continued to the $n$th observation without the potential prey item either attacking (if it is undesirable) or escaping (if it is desirable). Then

$$
\pi_{n}=\frac{(1-\Delta)^{n} p}{(1-\Delta)^{n} p+(1-\Xi)^{n}(1-p)},
$$

and

$$
\lambda_{n}=\frac{1}{2}-\sigma^{2}(n) \log \left(\left(\frac{1-\Delta}{1-\Xi}\right)^{n} \frac{p\left|v_{d}\right|}{(1-p)\left|v_{u}\right|}\right) .
$$

If the predator has made a single observation $X_{0}$, it now needs to determine what to do next: whether to attack immediately, flee immediately, or continue examining the potential prey item. In order to make this decision it needs to know the expected reward of making exactly $n$ additional observations before deciding whether to attack or flee. If the object is actually desirable, the probability it will 
not have escaped after $n$ observations is $(1-\Delta)^{n}$, and it will give the reward $v_{d}$ if the predator ultimately decides to attack, and 0 if it decides to flee or the prey escapes. If the object is actually undesirable, then the predator will survive to make the $n$ observations with probability $(1-\Xi)^{n}$, receiving the cost $v_{u}$ if it does not survive that long or if it ultimately decides to attack, and 0 otherwise. Thus the overall expected value is

$$
\begin{aligned}
& V_{n}=v_{d} P\left(D=1 \mid X_{0}\right)(1-\Delta)^{n} P\left(\bar{X}_{n}>\lambda_{n} \mid X_{0}, D=1\right)+ \\
& v_{u} P\left(D=0 \mid X_{0}\right)\left(\left(1-(1-\Xi)^{n}\right)+(1-\Xi)^{n} P\left(\bar{X}_{n}>\lambda_{n} \mid X_{0}, D=0\right)\right) .
\end{aligned}
$$

The probability that the predator will decide to attack, $P\left(\bar{X}_{n}>\lambda_{n} \mid X_{0}, D\right)$, depends on the distribution of the future information $X_{1}, \ldots, X_{n}$ that the predator will obtain. The expected value in the specific case of making no new observations and either attacking or fleeing immediately is given by

$$
V_{0}=\max \left\{0, v_{d} P\left(D=1 \mid X_{0}\right)+v_{u} P\left(D=0 \mid X_{0}\right)\right\},
$$

since $X_{0}>\lambda_{0}$ if and only if the expected reward of attacking is positive.

\subsubsection{Predicting future decisions}

Now suppose the predator has made observations $X_{0}, \ldots, X_{k}$, and must now predict what evidence it will have after it has made an additional $n-k$ observations, $X_{k+1}, \ldots, X_{n}$. First, it can determine what information it has on the true phenotype. A bit of algebra shows that

$$
T \mid \bar{X}_{k}, D \sim N\left(\frac{(k+1) \sigma_{p}^{2} \bar{X}_{k}+\sigma_{e}^{2} D}{(k+1) \sigma^{2}(k)}, \frac{\sigma_{p}^{2} \sigma_{e}^{2}}{(k+1) \sigma^{2}(k)}\right) .
$$


Then it follows that for $k<n, \bar{X}_{n}$ is normally distributed conditional on $\bar{X}_{k}$ and $D$, and

$$
\begin{aligned}
\mathbb{E}\left[\bar{X}_{n} \mid \bar{X}_{k}, D\right] & =\mathbb{E}\left[\frac{k+1}{n+1} \bar{X}_{k}+\frac{n-k}{n+1} T+\frac{\sum_{i=k+1}^{n}\left(X_{i}-T\right)}{n+1} \mid \bar{X}_{k}, D\right] \\
& =\frac{\sigma^{2}(n)}{\sigma^{2}(k)} \bar{X}_{k}+\frac{(n-k) \sigma_{e}^{2}}{(n+1)(k+1) \sigma^{2}(k)} D \\
\operatorname{Var}\left[\bar{X}_{n} \mid \bar{X}_{k}, D\right] & =\operatorname{Var}\left[\frac{k+1}{n+1} \bar{X}_{k}+\frac{n-k}{n+1} T+\frac{\sum_{i=k+1}^{n}\left(X_{i}-T\right)}{n+1} \mid \bar{X}_{k}, D\right] \\
& =\frac{(n-k) \sigma_{e}^{2}}{(n+1)(k+1) \sigma^{2}(k)} \sigma^{2}(n) .
\end{aligned}
$$

Then the probability that the predator will decide to attack the object, after having made the additional observations, is

$$
P\left(\bar{X}_{n}>\lambda_{n} \mid \bar{X}_{k}, D\right)=1-\Phi\left(\frac{\lambda_{n}-\mathbb{E}\left[\bar{X}_{n} \mid \bar{X}_{k}, D\right]}{\sqrt{\operatorname{Var}\left[\bar{X}_{n} \mid \bar{X}_{k}, D\right]}}\right),
$$

where $\Phi$ is the cumulative distribution function of the standard normal distribution.

\subsubsection{Existence of an optimum}

Once we can calculate the expected rewards $V_{n}$, based on the information contained in $X_{0}$ at least, the optimal strategy for the predator is to make $n^{*}$ additional observations, where $n^{*}$ is an integer maximizing $V_{n^{*}}$, and then to decide whether to attack or flee. Note, however, that this strategy is only optimal among those strategies in which the predator takes a fixed number of prey: it may be possible to do better with a flexible strategy where the number of observations is varied based on the outcome of the first observation. To prove that there is such an $n^{*}$, consider

$$
\lim _{n \rightarrow \infty} V_{n}=v_{u} P\left(D=0 \mid X_{0}\right)<V_{0} .
$$


Since the $V_{n}$ converge to a value strictly less than $V_{0}$, all but finitely many $V_{n}$ are less than $V_{0}$, and so there must be a maximum $V_{n}$. In fact, we can find an upper bound to the point at which $V_{n}$ becomes consistently less than $V_{0}$,

$$
n \geq-\frac{\log 2}{\log (1-\min (\Delta, \Xi))} \Longrightarrow V_{n} \leq V_{0},
$$

which provides a simple way to determine $n^{*}$, by calculating the finitely many $V_{n}$ up to this bound and finding the maximum (this upper bound is proven in the supplementary material).

\section{$3 \cdot 3$ Results}

The first decision facing a predator when it encounters a startle display is whether to make additional observations, or to immediately make a binding decision and attack or flee. The optimal decision in this first step can be determined from the $n^{*}$ value derived from the model. If $n^{*}>0$, then the expected reward is maximized for a non-zero number of additional observations, and the predator should continue to observe. If $n^{*}=0$, the predator should make a final decision immediately, either attacking if $V_{0}>0$, or fleeing otherwise. The results of this analysis, showing the optimal decision at various points in parameter space, are shown in Figures 3.1 and 3.2. specifically for a case where desirable objects are slightly more common the environment $(p=0.7$; cases for other $p$ are shown in the supplemental figures). Figure 3.1 shows the effect of varying initial observations, and Figure 3.2 shows the effect of varying $\Xi$ at a fixed, small $\Delta$ (a figure showing the converse, varying $\Delta$, is in the supplementary figures). Note that instead of directly showing $\sigma_{p}^{2}$ and $\sigma_{e}^{2}$, the figures show the standard deviations of individual observations $\sigma(0)=\sqrt{\operatorname{Var}\left[X_{i} \mid D\right]}=\sqrt{\sigma_{p}^{2}+\sigma_{e}^{2}}$, and the correlation between the observations $r=\operatorname{Cor}\left[X_{i}, X_{j} \mid D\right]=\frac{\sigma_{p}^{2}}{\sigma_{p}^{2}+\sigma_{e}^{2}}$. These two quantities are interpretable as measures of 
the difficulty in distinguishing the two classes of potential prey (since the means of the two classes are fixed at 0 and 1, lower standard deviations imply higher discriminability) and the relative importance of phenotypic variation over error variation respectively.

The underlying signal detection model for single observations is visible in Figure 3.1 in the distribution of cases where the optimal strategy is to attack or flee: there is a threshold at an intermediate observed phenotype below which it is best to attack and above which it is best to flee. Now, however, there is an additional option to continue making observations in many cases, which adds another threshold, so that the optimal strategy goes from fleeing to observing further to attacking as $X_{0}$ increases. Continuing is favoured when there is less total (both error and phenotypic) variability in perceived signals (low values of $\sigma^{2}(0)$ ), when error variability is more important than phenotypic variability (low $r$ ), when undesirable potential prey are less likely to attack (low $\Xi$ ) and desirable prey less likely to escape (low $\Delta$ ), and for values of the cost/benefit ratio closer to the threshold.

For more detail we can examine the actual values of $n^{*}$. In most cases it is fairly small, always less than five, with high values favoured only when the probability of losing the potential prey item through escape or being attacked by it during further examination is very low.

\subsection{A second model: distance}

As an alternative to the model in the previous sections, suppose that the predator has the capacity to choose the distance at which it makes a subsequent observation of the potential prey item. Then it clearly faces a trade-off: moving further away reduces the risk of being attacked by an ambush predator, but increases the probability of an actual prey item escaping, and reduces the amount of informa- 


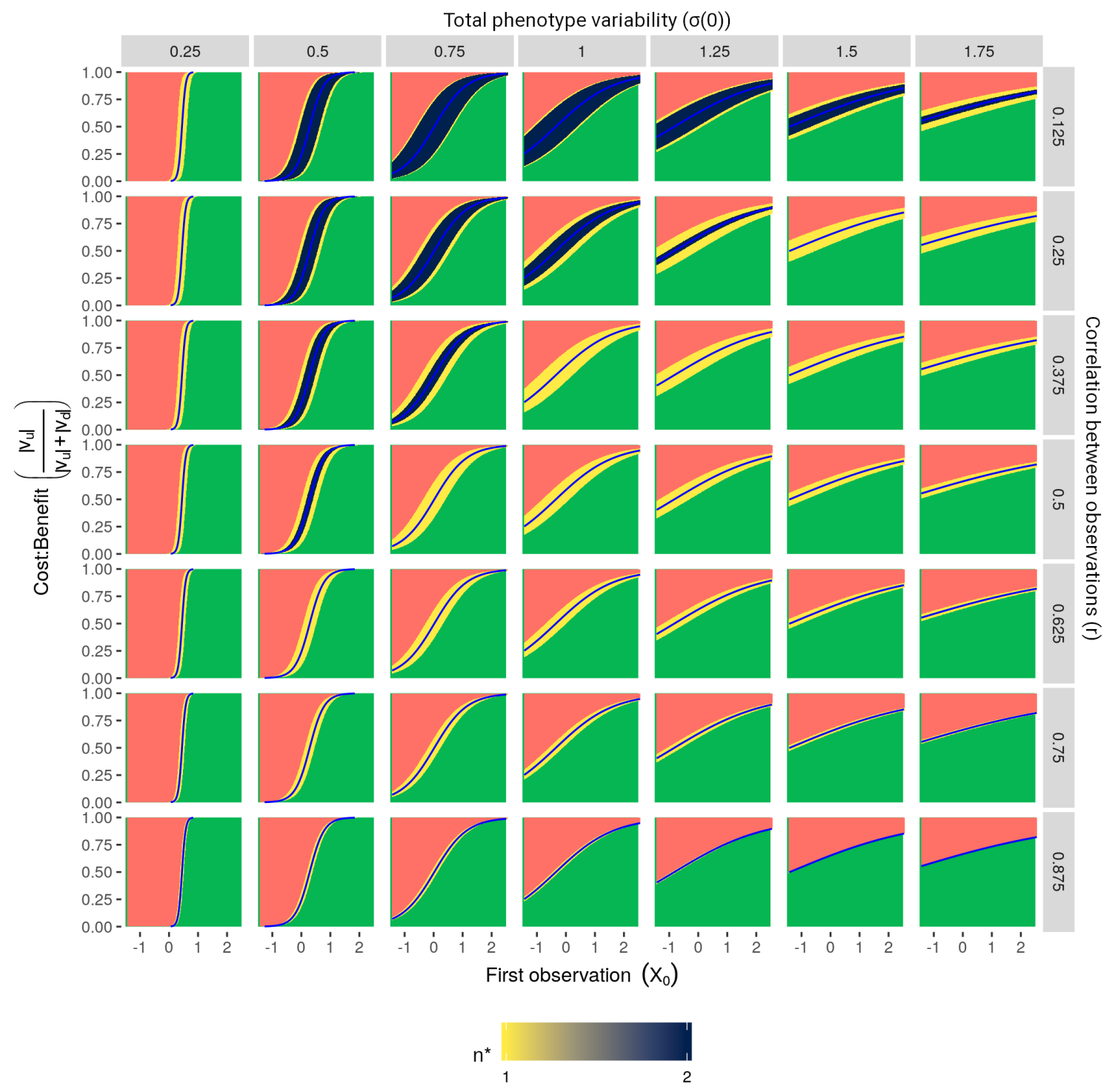

Figure 3.1: The optimal action after having made a single observation of the prey, at a background frequency of desirable prey of $p=0.7$ and with $\Delta=0.05=\Xi$. The optimal action is either to attack immediately (green area of each panel), flee immediately (red area), or continue observing for $n^{*}$ additional observations (coloured by $n^{*}$, as in legend). Columns correspond to values of $\sigma(0)=\sqrt{\sigma_{p}^{2}+\sigma_{e}^{2}}$, the standard deviation of a single observation, and rows to $r=\frac{\sigma_{p}^{2}}{\sigma_{p}^{2}+\sigma_{e}^{2}}$, the correlation between individual observations. Observations are more characteristic of undesirable objects for lower values of $X_{0}$ : undesirable objects have an average phenotype of 0 , and desirable objects of 1 . The blue line indicates the attack-flee threshold of a single step signal detection model. 


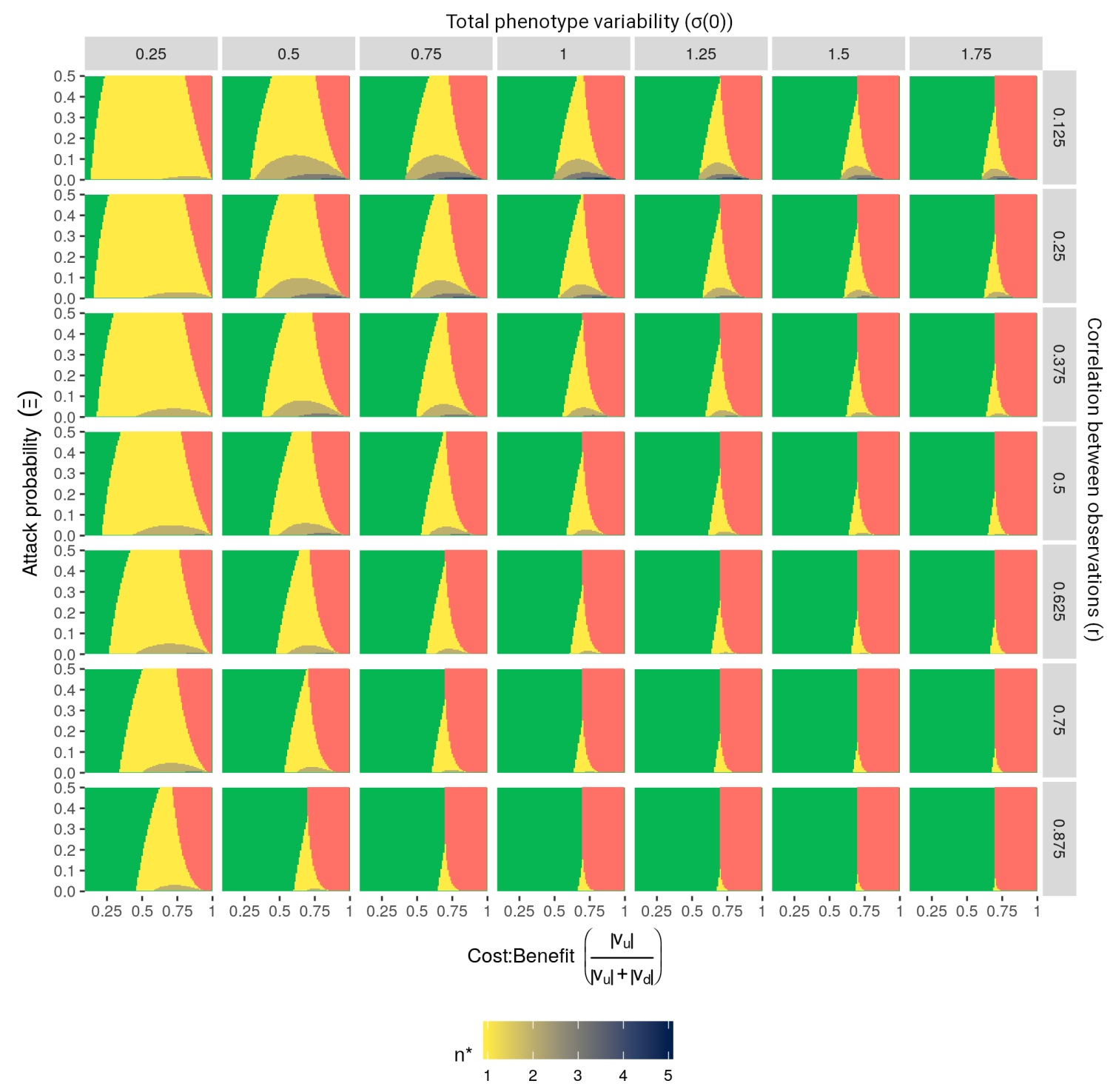

Figure 3.2: The optimal action after having made a single observation of the prey $X_{0}=0.5$, at a background frequency of desirable prey of $p=0.7$ and with $\Delta=0.05$. The optimal action is either to attack immediately (green area of each panel), flee immediately (red area), or continue observing for $n^{*}$ additional observations (coloured by $n^{*}$, as in legend). Columns correspond to values of $\sigma(0)=\sqrt{\sigma_{p}^{2}+\sigma_{e}^{2}}$, the standard deviation of a single observation, and rows to $r=\frac{\sigma_{p}^{2}}{\sigma_{p}^{2}+\sigma_{e}^{2}}$, the correlation between individual observations. 
tion from additional samples, because the perceptual error increases. This can be incorporated into the model by making the parameters $\Xi, \Delta$, and $\sigma_{e}^{2}$ dependent on the distance $d$ from the potential prey item:

$$
\Xi(d)=\frac{1}{1+\xi d} \quad \Delta(d)=\frac{\delta d}{1+\delta d} \quad \sigma_{e}^{2}(d)=\epsilon^{2} d .
$$

The last of these is based on the example of a visual predator with constant angular resolution of their vision: the minimum distinguishable distance, and therefore the perceptual error, is then proportional to distance. The first encounter with the potential prey item is made at a distance of 1 (we can make this assumption without loss of generality because the units of distance are arbitrary). As before, the predator may immediately commit to attacking or fleeing, or make an additional observation first. Now however, it may choose the distance from which the additional observation will be made. The optimum distance is the value of $d$ maximizing

$$
\begin{aligned}
& v_{d} P\left(D=1 \mid X_{0}\right)(1-\Delta(d)) P\left(\bar{X}>\lambda \mid X_{0}, D=1\right)+ \\
& \quad v_{u} P\left(D=0 \mid X_{0}\right)\left(\Xi(d)+(1-\Xi(d)) P\left(\bar{X}>\lambda \mid X_{0}, D=0\right)\right),
\end{aligned}
$$

where

$$
\lambda=\frac{1}{2}-\left(\sigma_{p}^{2}+\frac{\sigma_{e}^{2}(1)+\sigma_{e}^{2}(d)}{4}\right) \log \frac{(1-\Delta(d)) p\left|v_{d}\right|}{(1-\Xi(d))(1-p)\left|v_{u}\right|},
$$

and $\bar{X}$, the mean of the two observations, has a normal distribution conditional on $X_{0}$ and $D$, with

$$
\begin{aligned}
\mathbb{E}\left[\bar{X} \mid X_{0}, D\right] & =\frac{2 \sigma_{p}^{2}+\sigma_{e}^{2}(1)}{2\left(\sigma_{p}^{2}+\sigma_{e}^{2}(1)\right)} X_{0}+\frac{\sigma_{e}^{2}(1)}{2\left(\sigma_{p}^{2}+\sigma_{e}^{2}(1)\right)} D \\
\operatorname{Var}\left[\bar{X} \mid X_{0}, D\right] & =\frac{1}{4}\left(\frac{\sigma_{p}^{2} \sigma_{e}^{2}(1)}{2\left(\sigma_{p}^{2}+\sigma_{e}^{2}(1)\right)}+\sigma_{e}^{2}(d)\right) .
\end{aligned}
$$




\subsubsection{Results}

The optimal distance from which to make a single additional observation is shown in Figures 3.3 and 3.4 . The effect of altering the dependence of $\Xi(d)$ on distance is shown in Figure 3.4 (larger $\xi$ corresponds to $\Xi(d)$ decreasing more quickly with distance; a similar figure for $\Delta(d)$ is in the supplementary figures). The figures show $\sigma(0)$ and $r$ at the time of the initial observation to remain comparable to the figures in the previous section, but of course in this model there is not a single variance and correlation of observations: these are now dependent on the distance. As in the previous model, the underlying signal detection pattern, with a single optimal threshold, is visible in the pattern of immediate decisions, but with an overlayed area where observing again at a distance is optimal. Intriguingly, it is far more common for the optimal strategy to be to move closer to make an additional observation: more distant observation is optimal only at the very edge of the region where an additional observation is favoured.

\subsection{Discussion}

Predators encountering a startle display are faced with a trade-off between two possible costs: harm that could be inflicted by a danger such as an ambush predator, and the lost opportunity of abandoning a good prey item. In trying to identify which of the two classes a particular potential prey item falls into, the predator also faces a speed-accuracy trade-off. The former, cost-benefit trade-off is modelled with signal detection theory; the model presented here extends signal detection to also include the latter speed-accuracy trade-off by incorporating the time taken to examine an object before making a decision in the form of the number of observations made. The distance-based model also extends signal detection theory to 


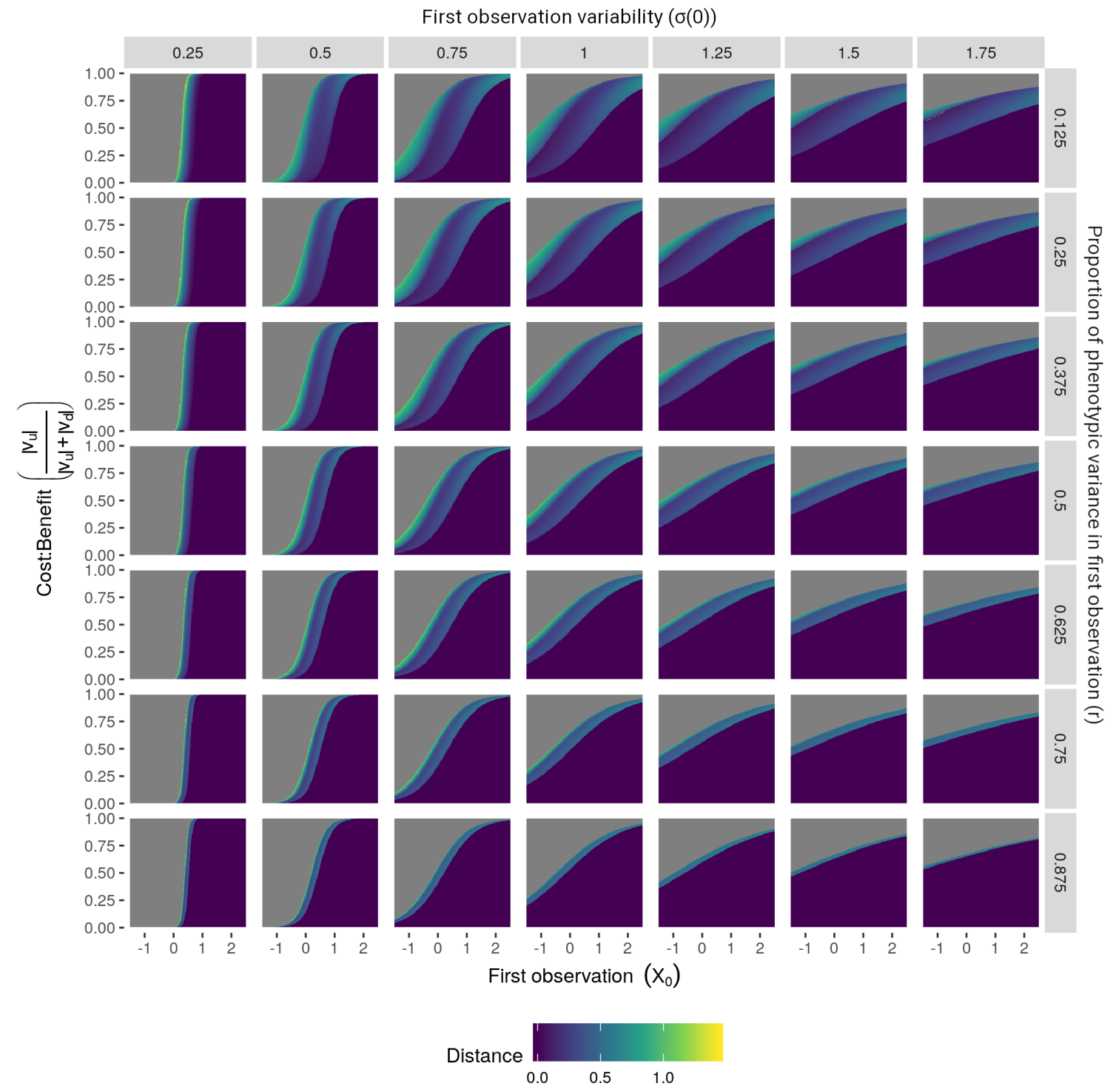

Figure 3.3: The optimal distance $d$ to make a single additional observation of the prey. Grey indicates $d=\infty$, i.e. fleeing immediately, and $d=0$ (dark purple) indicates attacking immediately. The background frequency of desirable prey is fixed at $p=0.7$ and the attack and escape probability coefficients at $\xi=0.05=\xi$. Columns correspond to values of $\sigma(0)=\sqrt{\sigma_{p}^{2}+\sigma_{e}^{2}(1)}=\sqrt{\sigma_{p}^{2}+\epsilon^{2}}$, the standard deviation of the initial observation at $d=1$, and rows to $r=\frac{\sigma_{p}^{2}}{\sigma_{p}^{2}+\sigma_{e}^{2}(1)}=\frac{\sigma_{p}^{2}}{\sigma_{p}^{2}+\epsilon^{2}}$. 
First observation variability $(\sigma(0))$

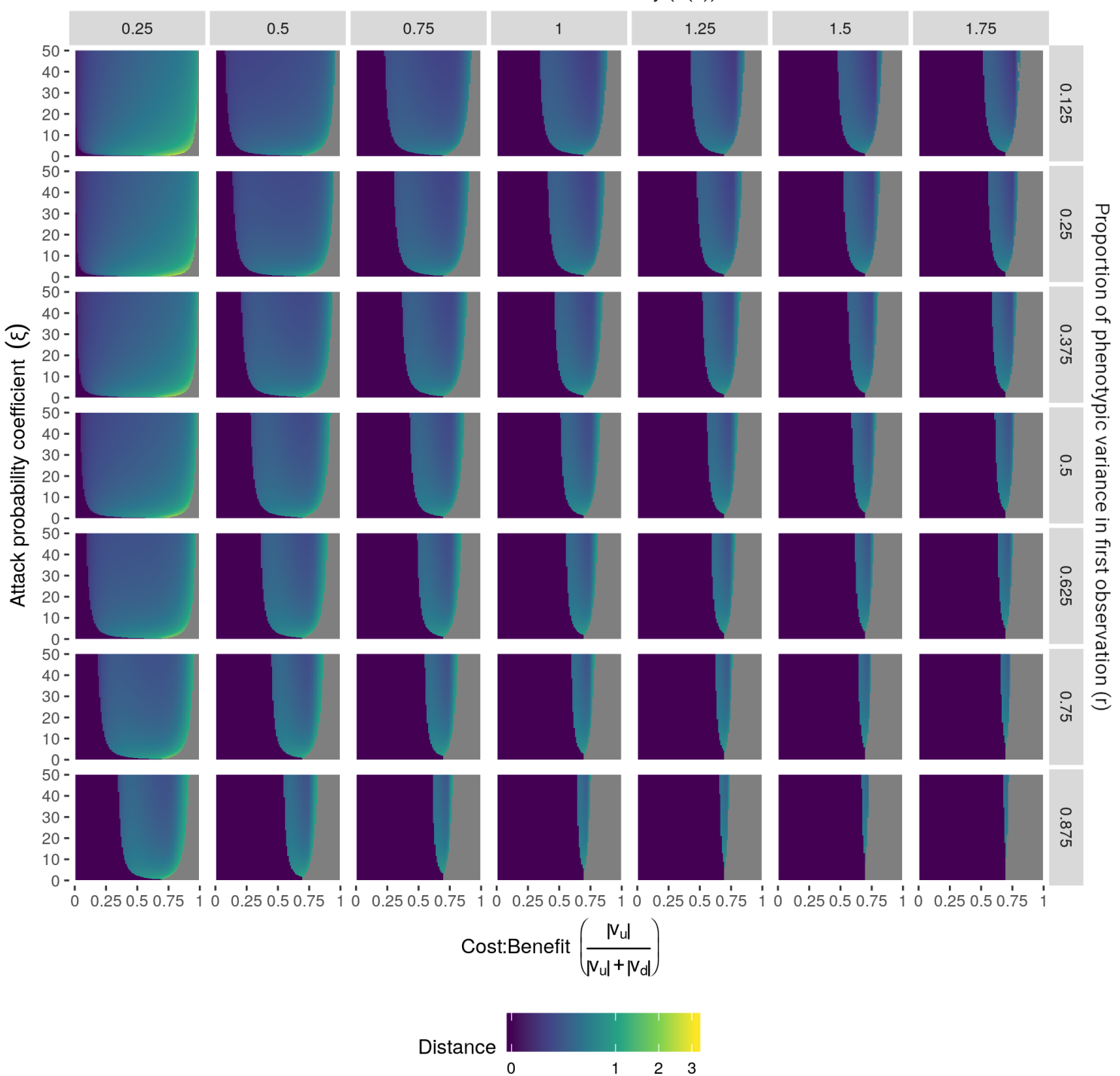

Figure 3.4: The optimal distance $d$ to make a single additional observation after having made an observation $X_{0}=0.5$ at a distance of 1 . Grey indicates $d=\infty$, i.e. fleeing immediately, and $d=0$ (dark purple) indicates attacking immediately. The background frequency of desirable prey is fixed at $p=0.7$ and the escape probability coefficient at $\delta=0.05$. Columns correspond to values of $\sigma(0)=$ $\sqrt{\sigma_{p}^{2}+\sigma_{e}^{2}(1)}=\sqrt{\sigma_{p}^{2}+\epsilon^{2}}$, the standard deviation of the initial observation at $d=1$, and rows to $r=\frac{\sigma_{p}^{2}}{\sigma_{p}^{2}+\sigma_{e}^{2}(1)}=\frac{\sigma_{p}^{2}}{\sigma_{p}^{2}+\epsilon^{2}}$. 
include a trade-off between safety and accuracy.

A key component of both models is the separation of the variability in observations into two components, the inherent variability in phenotypes in the population and the variability between observations of the same individual due to perceptual error. The inclusion of the latter variability enables us to model the gain of information from repeated observations, and in the distance model the reduced information available when the predator moves farther away. However, the former variation is a lower bound on the variability, below which it cannot be eliminated by sampling. This separation of variability has been used before in models of speed-accuracy trade-offs (Abbott and Sherratt 2013), but here we combine it with a novel cost structure based on startle displays: the cost of sampling comes from the examined object (through the possibility of either being attacked or losing a good prey item), and is distinct for the two classes of potential prey items. A previous model, the "Gruffalo" model of Castellano and Cermelli (2015), did include costs of sampling, but from the threat of predation from another predator, not from the risk of the examined object itself attacking while being examined. This further means that the length of time that the predator is able to examine the potential prey item is a source of information, which is incorporated through the factor of $\left(\frac{1-\Delta}{1-\Xi}\right)^{n}$ incorporated into $\lambda_{n}$.

Although developed for startle displays, the modelling framework extends in general to combinations of speed-accuracy and signal detection trade-offs where the cost of sampling comes from one or both of the potential classes of stimuli. For example, even in operations research where signal detection theory was developed, a blip on the radar could be a whale or an enemy submarine, and a quick avoidance response may be favoured over further investigation because the latter case is so dangerous (Marcum 1947). Likewise decisions following inspection of 
medical images are rarely binary, and often involve situations where rapid treatment of affected patients is important to beneficial outcomes.

Obviously, making optimal decisions about potential prey items is advantageous for predators; but the results of the model can also be interpreted from the perspective of the prey, as showing under what circumstances startle displays are an effective anti-predator defence. In order to be effective, the startle display must cause the predator to break off, or at least delay, an attack, giving the prey time to flee. Figures 3.1 and 3.2 show that there is a large area of the parameter space in which it is optimal for predators to delay attacks or flee. In general, it is advantageous to continue examining when that course of action is likely to provide useful information, either because desirable and undesirable objects are sufficiently distinct or because perceptual error contributes more than phenotypic variability to the variance, and when the risks of sampling are low. The range of acceptable $\Xi$ and $\Delta$ for an immediate attack or flight is widest close to the threshold of the underlying simple signal detection model, where continued examination is most useful to tell desirable and undesirable objects apart, whereas for more extreme values of the cost/benefit ratio, the range is narrow. Figures 3.3 and 3.4 show that in the model which includes distance, the range of parameter space in which it is advantageous to delay is similar, if not larger, although for prey this comes at the cost of the predator usually moving closer (and thus making escape harder) during the delay.

One notable aspect of the results is that continuing to examine is most broadly favoured for low values of $\Xi$ and of $\Delta$, where the risk of losing an opportunity or being attacked is low, making continued examination low-risk. Figure 3.2 also shows that the optimal delays are longest for these low values of $\Xi$, and Figure D.1 similarly for $\Delta$. This suggests that in circumstances where the cost/benefit ratio 
is low, it is advantageous for a startle display to be confused for a less dangerous model in order to encourage the predator to delay rather than take a chance (when the cost/benefit ratio is high, it is still better to be confused for a high $\Xi$ model, because the predator is more likely to flee outright). In other words, it is best to be confused for something with high cost and high $\Xi$, but when the prey is confused for something with low cost, it is better to be confused for something unlikely to attack an observing predator (i.e. with low $\Xi$ ).

The model, although it describes predator responses to startle displays, doesn't explicitly contain any element of sudden exposure or change in phenotype: it could be applied equally to a constantly visible signal. The advantage of the startle component comes from a variety of sources. Suddenly displaying the signal ensures that the predator is close enough when it begins to examine the prey that the prey is aware of its presence and can use the opportunity to escape: if the predator could examine the prey to its satisfaction and then attack without being detected, the display would offer no defence. As well, the sudden exposure of the startle display produces a confusion effect, making the display harder for the predator to clearly interpret, and increasing the resemblance of the display to an attack; in terms of the model, this corresponds to increasing $\sigma_{e}^{2}$, as the sudden appearance reduces the precision of observation, and, most importantly, increasing the predator's estimate of $\Xi$, as the potential prey item seems more likely to imminently attack. The startle thus increases the efficacy of the underlying signal in producing the desired outcome for the prey (Guilford and Dawkins 1991). Additionally, the startle display may exist as a component of a portfolio of successive defences (Kang et al. 2016; Bateman et al. 2014; Britton et al. 2007), where being suddenly revealed allows it to act as a backup to another defence. A startle display is a low cost defence that can be deployed early following encounter, and it is particularly 
suitable as a backup defence to crypsis (Umbers et al. 2017).

Our model focuses on a single encounter, but there are also aspects of startle displays that are observed over multiple encounters, particularly the desensitization (habituation) of predators to startle displays when they are encountered frequently (Dookie et al. 2017, Kang et al. 2017). At least in part, this phenomenon can be explained in terms of pooling observations over multiple encounters, which would increase the effective number of samples made, with reduced risk. Equivalently, this would have the effect of reducing $\sigma_{e}^{2}$ in each encounter, provided the predator encounters the particular prey often enough. However, the effectiveness of this learning process, and thus the importance of habituation, may be limited by the startle display itself, if predators flee or the prey escapes before the predator learns whether the prey was a desirable prey item or not; a similar mechanism has been proposed to be responsible for the evolution of imperfect mimicry (Chittka and Osorio 2007; Sherratt and Peet-Paré 2017). This would also mean that habituation would likely be more prominent in experiments than in the wild: in an experimental situation, the predator is constrained to see the startle display a certain number of times or for a certain length of time, no matter what choice it would rather make (Dookie et al.2017, Kang et al.2017), and it may gain enough experience to be habituated, while in the wild it would flee early in every encounter, and never gain enough experience with the startle display. The possibility in the real world to encounter multiple prey items also means that examination of the prey item has an opportunity cost for the predator, which could be looking for more obviously good prey items instead of spending time examining the same prey item carefully. This would have the most impact in the small area of the parameter space where $n^{*}$ is high. Extensions of signal detection theory to include opportunity costs were presented by Abbott and Sherratt (2013) and Holen (2013), and 
could be combined with the present model.

Startle displays have obvious advantages to the prey that use them as antipredator defences, in the form of an increased survival rate in encounters (Olofsson et al. 2012; Kang et al. 2017). However, being startled is also an adaptive response of predators that want to deal with an uncertain situation correctly but also quickly. The interaction of two trade-offs, false positive against false negatives (the classic signal detection trade-off) and speed against accuracy, should be included in a suitable model of the optimal decision making of predators. The decisions of predators in turn provide the opportunity for startle displays to be effective. 


\section{CONCLUSION}

... when the mind is in its infancy a Bias is in reality a

Bias, but when we have acquired more strength, a

Bias becomes no Bias.

John Keats, letter to J. H. Reynolds, 3 May 1818

In this thesis, I have presented two models of optimal decision making by predators faced with uncertainty about the available prey. In the first, I developed a model framework to describe predators learning about the potential prey in their environment in a Bayesian fashion, and then applying that knowledge to optimal decision making. The model goes beyond merely reproducing the empirical characteristics of generalization to focus on the rational and adaptive significance of generalization as a learning process. In the second, I developed a model of a specific situation, a predator facing a startle display. This involved an extension of classical signal detection theory to include speed-accuracy trade-offs, by incorporating the possibility of continued examination, in addition to the classical accept and reject options available in signal detection models.

Although the models both involve predator behaviour and uncertainty about the suitability of a potential prey item, they are quite different models, both mathematically and biologically, and deal with quite distinct problems using very different methods. However, there are a few commonalities to comment upon here. The first is that they are both in some sense models of optimal behaviour: they both deal with predators that act to maximize the expected fitness outcome of 
their decisions. Although optimization models are well established in behavioural ecology, they must be interpreted carefully, as real organisms do not necessarily always behave optimally (see Krebs and Kacelnik (1991) for an overview of objections and responses to optimality in modelling). An optimization model is perhaps better thought of as a model of the adaptive landscape of behaviour than its actual realization. Nonetheless, selection ensures that actual behaviour is generally a good approximation to the optimal behaviour.

Another similarity between the two models is that they both involve partitioning uncertainty or variability into multiple components. In the startle model, this is the division of the variation in observations of prey into inherent variation between individuals $\sigma_{p}^{2}$ and perceptual error $\sigma_{e}^{2}$. In the generalization model, uncertainty about a prey item comes from both the actual variability in the environment and the predator's uncertainty about the distribution of prey; this is modelled by having a probability distribution (the subjective or epistemic uncertainty of the predator) over the possible distributions of prey in the environment (the objective or aleatory uncertainty in any randomly selected prey item). This latter combination of subjective and objective uncertainty is particularly common in a variety of applications of Bayesian probability, and the interaction between the two types of probabilities is described by the "principal principle" of Lewis $(1980)$. This kind of separation of multiple sources of uncertainty is in general a very useful idea when building models that have a statistical component. Fennell and Baddeley (2012) have even applied it to modelling deviations of behaviour from expected utility maximization.

There are also potential extensions that the two models have in common. One of these is the incorporation of opportunity costs or state-dependent foraging into the model. This would involve adjusting the payoffs of the model to account for 
the fact that the costs and benefits are measured relative to the condition of the predator, and the other options for foraging it has (Sherratt 2003). In addition to including the effect of the environmental state on decisions, this could include extending the models to sequences of many encounters with prey. Ultimately, this could lead to models of the evolution of anti-predator defences. Finally, it might be possible to extend the two models by combining them. The simple normal-normal model of signal detection theory could be replaced with a Bayesian model of generalization. The predator could even estimate the other parameters of the startle model based on its experience using the generalization model: for example rather than have one $\Xi$ for all prey, it could be estimated for the individual being examined based on generalization from previous interactions with potential ambush predators and the information on phenotype being discovered. 


\section{REFERENCES}

Abbott, K. R. and T. N. Sherratt (2013). "Optimal sampling and signal detection: unifying models of attention and speed-accuracy trade-offs". In: Behavioral Ecology 24.3, pp. 605-616. Dor: 10.1093/beheco/artoo1

Arnold, S. J. (1978). "The evolution of a special class of modifiable behaviors in relation to environmental pattern". In: The American Naturalist 112.984, pp. 415427. DOI: $10.1086 / 283283$.

Aubier, T. G., M. Joron, and T. N. Sherratt (2017). "Mimicry among unequally defended prey should be mutualistic when predators sample optimally". In: The American Naturalist 189.3, pp. 267-282. Dor: 10.1086/690121.

Baddeley, R. J., D. Osorio, and C. D. Jones (2007). "Generalization of color by chickens: experimental observations and a Bayesian model". In: The American Naturalist 169 , S27-S41. Dor: $10.1086 / 510142$

Balogh, A. C. V., G. Gamberale-Stille, B. S. Tullberg, and O. Leimar (2010). "Feature theory and the two-step hypothesis of Müllerian mimicry evolution". In: Evolution 64.3, pp. 810-822. Dor: $10.1111 / \mathrm{j} \cdot 1558-5646.2009 \cdot 00852 \cdot \mathrm{x}$.

Balogh, A. C. V. and O. Leimar (2005). "Müllerian mimicry: an examination of Fisher's theory of gradual evolutionary change". In: Proceedings of the Royal Society B 272.1578, pp. 2269-2275. Dor: 10.1098/rspb.2005.3227.

Barnett, C. A., M. Bateson, and C. Rowe (2007). "State-dependent decision making: educated predators strategically trade off the costs and benefits of consum- 
ing aposematic prey". In: Behavioral Ecology 18.4, pp. 645-651. DoI: 10.1093/ beheco/armo27.

Barrett, J. A. (1976). "The maintenance of non-mimetic forms in a dimorphic Batesian mimic species". In: Evolution 30.1, pp. 82-85. Dor: $10 \cdot 1111 /$ j $1558-$ $5646 \cdot 1976 \cdot \mathrm{tboo88} 3 \cdot \mathrm{x}$.

Bateman, A. W., M. Vos, and B. R. Anholt (2014). "When to defend: antipredator defenses and the predation sequence". In: The American Naturalist 183.6, pp. $847-855$. DOI: $10.1086 / 675903$.

Bates, H. W. (1862). "Contributions to an insect fauna of the Amazon valley. Lepidoptera: Heliconidae". In: Transactions of the Linnean Society of London 23.3, pp. 495-566. Dor: $10.1111 / \mathrm{j} .1096-3642 \cdot 1860$. tboo $146 \cdot x$

Blanchard, B. D. and C. S. Moreau (2016). "Defensive traits exhibit an evolutionary trade-off and drive diversification in ants". In: Evolution 71.2, pp. 315-328. DoI: $10.1111 / \mathrm{evo} .13117$

Bobisud, L. E. (1978). "Optimal time of appearance of mimics". In: The American Naturalist 112.987, pp. 962-965. DoI: $10.1086 / 283338$.

Bobisud, L. E. and C. J. Potratz (1976). "One-trial versus multi-trial learning for a predator encountering a model-mimic system". In: The American Naturalist 110.971, pp. 121-128. DOI: $10.1086 / 283052$.

Bowers, M. D. (1992). "The evolution of unpalatability and the cost of chemical defense in insects". In: Insect Chemical Ecology: An Evolutionary Approach. Ed. by B. N. Roitberg and M. B. Isman. New York: Chapman \& Hall. Chap. 7, pp. $216-$ 244 .

Britton, N. F., R. Planqué, and N. R. Franks (2007). "Evolution of defence portfolios in exploiter-victim systems". In: Bulletin of Mathematical Biology 69.3, pp. 957988. DoI: $10.1007 / \mathrm{s} 11538-006-9178-5$. 
Brockhurst, M. A., T. Chapman, K. C. King, J. E. Mank, S. Paterson, and G. D. D. Hurst (2014). "Running with the Red Queen: the role of biotic conflicts in evolution". In: Proceedings of the Royal Society B 281.1797, p. 20141382. Dor: $10.1098 / \mathrm{rspb} .2014 \cdot 1382$.

Broom, M., A. D. Higginson, and G. D. Ruxton (2010). “Optimal investment across different aspects of anti-predator defences". In: Journal of Theoretical Biology 263.4, pp. 579-586. Dor: $10.1016 / \mathrm{j} \cdot \mathrm{jtbi} .2010 .01 .002$.

Carpenter, B., A. Gelman, M. Hoffman, D. Lee, B. Goodrich, M. Betancourt, M. Brubaker, J. Guo, P. Li, and A. Riddell (2017). "Stan: a probabilistic programming language”. In: Journal of Statistical Software 76.1, pp. 1-32. Dor: 10. 18637/ jss.v076.io1.

Castellano, S. (2019). "Putting mechanisms in foraging theory: the role of computational mechanisms in optimal decision making". In: Animal Behaviour 153 , pp. 159-169. DOI: 10.1016/ j . anbehav.2019.04.015.

Castellano, S. and P. Cermelli (2015). “Preys' exploitation of predators' fear: when the caterpillar plays the Gruffalo". In: Proceedings of the Royal Society B 282, p. 20151486 . DOI: $10.1098 / \mathrm{rspb} .2015 \cdot 1786$.

Chatera, N. and P. M. Vitányi (2003). "The generalized universal law of generalization”. In: Journal of Mathematical Psychology 47.3, pp. 346-369. Dor: 10.1016/ S0022-2496(03)00013-0.

Cheng, K. (2000). "Shepard's universal law supported by honeybees in spatial generalization". In: Psychological Science 11.5, pp. 403-408. Dor: 10 . 1111/14679280.00278 .

- (2002). "Generalisation: mechanistic and functional explanations". In: Animal Cognition 5.1, pp. 33-40. DOI: 10.1007/s10071-001-0122-7. 
Cheng, K. and M. L. Spetch (2002). "Spatial generalization and peak shift in humans". In: Learning and Motivation 33.3, pp. 358-389. Dor: $10.1016 /$ Soo239690( 02$) 00003-6$.

Cheng, K., M. L. Spetch, and M. Johnston (1997). "Spatial peak shift and generalization in pigeons". In: Journal of Experimental Psychology: Animal Behavior Processes 23.4, pp. 469-481. DoI: 10.1037/0097-7403.23.4.469.

Chittka, L. and D. Osorio (2007). "Cognitive dimensions of predator responses to imperfect mimicry". In: PLOS Biology 5.12, e339. Dor: 10.1371/ journal .pbio. 0050339 .

Chittka, L., P. Skorupski, and N. E. Raine (2009). "Speed-accuracy tradeoffs in animal decision making". In: Trends in Ecology \& Evolution 24.7, pp. 400-407. DOI: $10.1016 / \mathrm{j}$.tree.2009.02.010.

Cipollini, D., D. Waters, and C. Voelckel (2014). "Costs of resistance in plants: from theory to evidence". In: Insect-Plant Interactions. Ed. by C. Voelckel and G. Jander. Annual Plant Reviews 47. Wiley-Blackwell. Chap. 8, pp. 263-308. DOI: $10.1002 / 9781119312994 \cdot$ apro512.

De Bona, S., J. K. Valkonen, A. López-Sepulcre, and J. Mappes (2015). "Predator mimicry, not conspicuousness, explains the efficacy of butterfly eyespots". In: Proceedings of the Royal Society B 282.1806, p. 20150202 . DOI: $10.1098 /$ rspb. $2015 \cdot 0202$,

Dittrich, W., F. Gilbert, P. Green, P. McGregor, and D. Grewcock (1993). "Imperfect mimicry: a pigeon's perspective". In: Proceedings of the Royal Society B 251.1332, pp. 195-200. DoI: 10.1098/rspb.1993.0029.

Dookie, A. L., C. A. Young, G. Lamothe, L. A. Schoenle, and J. E. Yack (2017). "Why do caterpillars whistle at birds? Insect defence sounds startle avian predators". In: Behavioural Processes 138, pp. 58-66. Dor: $10.1016 / \mathrm{j}$. beproc.2017.02.002. 
Edmunds, M. (1974). Defence in Animals: A Survey of Anti-predator Defences. New York: Longman.

Edwards, W. (1965). "Optimal strategies for seeking information: models for statistics, choice reaction times, and human information processing". In: Journal of Mathematical Psychology 2.2, pp. 312-329. Dor: 10 . 1016/0022-2496(65) 90007-6.

Endler, J. A. (1991). "Interactions between predators and prey". In: Behavioural Ecology: An Evolutionary Approach. Ed. by J. R. Krebs and N. B. Davies. 3rd ed. Oxford: Blackwell Scientific Publishing. Chap. 6, pp. 169-202.

Estabrook, G. F. and D. C. Jespersen (1974). "Strategy for a predator encountering a model-mimic system". In: The American Naturalist 108.962, pp. 443-457. Dor: $10.1086 / 282925$

Fawcett, T. W. and R. A. Johnstone (2003). "Optimal assessment of multiple cues". In: Proceedings of the Royal Society B 270.1524, pp. 1637-1643. Dor: 10.1098/ rspb.2003.2328.

Fennell, J. and R. J. Baddeley (2012). "Uncertainty plus prior equals rational bias: an intuitive Bayesian probability weighting function." In: Psychological Review 119.4, pp. 878-887. Dor: 10.1037/a0029346.

Fisher, R. A. (1927). "On some objections to mimicry theory; statistical and genetic". In: Transactions of the Royal Entomological Society of London 75, pp. 269278.

Franks, D. W. and J. Noble (2003). "Batesian mimics influence mimicry ring evolution". In: Proceedings of the Royal Society B 271, pp. 191-196. Dor: 10.1098/ rspb.2003.2582, 
Franks, D. W. and J. Noble (2004). "Warning signals and predator-prey coevolution". In: Proceedings of the Royal Society B 271.1550, pp. 1859-1865. Dor: $10.1098 / \mathrm{rspb} .2004 .2795$

Franks, D. W., G. D. Ruxton, and T. N. Sherratt (2009). "Warning signals evolve to disengage Batesian mimics". In: Evolution 63.1 , pp. 256-267. Dor: $10.1111 / \mathrm{j}$. $1558-5646.2008 .00509 \cdot x$.

Franks, D. W. and T. N. Sherratt (2007). "The evolution of multicomponent mimicry". In: Journal of Theoretical Biology 244, pp. 631-639. Dor: 10.1016/ j . jtbi . 2006.09 .019 .

Froment, A. J. de, D. I. Rubenstein, and S. A. Levin (2014). "An extra dimension to decision-making in animals: the three-way trade-off between speed, effort per-unit-time and accuracy". In: PLoS Computational Biology 10.12, e1003937. Dor: $10.1371 /$ journal.pcbi.1003937.

Gamberale-Stille, G., A. C. V. Balogh, B. S. Tullberg, and O. Leimar (2012). "Feature saltation and the evolution of mimicry". In: Evolution 66.3, pp. 8o7-817. DOI: $10.1111 / \mathrm{j} \cdot 1558-5646.2011 .01482 \cdot \mathrm{x}$

Gamberale, G. and B. S. Tullberg (1996). "Evidence for a peak-shift in predator generalization among aposematic prey". In: Proceedings of the Royal Society B 263.1375, pp. 1329-1334. Dor: 10.1098/rspb.1996.0195.

Gavrilets, S. and A. Hastings (1998). "Coevolutionary chase in two-species systems with applications to mimicry". In: Journal of Theoretical Biology 191.4, pp. 415427. DOr: $10.1006 / \mathrm{jtbi} .1997 \cdot 0615$.

Gendron, R. P. and J. E. R. Staddon (1983). "Searching for cryptic prey: the effect of search rate". In: The American Naturalist 121.2, pp. 172-186. Dor: 10.1086/ 284049 . 
Getty, T. (1985). "Discriminability and the sigmoid functional response: how optimal foragers could stabilize model-mimic complexes". In: The American Naturalist 125.2, pp. 2239-256. DOI: $10.1086 / 284339$.

- (1996). "Mate selection by repeated inspection: more on pied flycatchers". In: Animal Behaviour 51.4, pp. 739-745. Dor: 10.1006/anbe.1996.0078.

Ghirlanda, S. and M. Enquist (2003). "A century of generalization". In: Animal Behaviour 66.1, pp. 15-36. Dor: $10.1006 /$ anbe.2003.2174.

Green, D. M. and J. A. Swets (1966). Signal Detection Theory and Psychophysics. New York: John Wiley and Sons.

Guilford, T. and M. S. Dawkins (1991). "Receiver psychology and the evolution of animal signals". In: Animal Behaviour 42.1, pp. 1-14. Dor: 10.1016/s0003$3472(05) 80600-1$.

Hadeler, K. P., P. de Mottoni, and A. Tesei (1982). "Mimetic gain in Batesian and Müllerian mimicry". In: Oecologia 53.1, pp. 84-92. Dor: $10.1007 / \mathrm{BF} 00377140$.

Halpin, C. G. and C. Rowe (2010). "Taste-rejection behaviour by predators can promote variability in prey defences". In: Biology Letters 6.5, pp. 617-619. DoI: $10.1098 / \mathrm{rsbl} .2010 .0153$

Halpin, C. G., J. Skelhorn, and C. Rowe (2014). "Increased predation of nutrientenriched aposematic prey". In: Proceedings of the Royal Society B 281.1781, p. 20133255 . DOI: $10.1098 / \mathrm{rspb} .2013 \cdot 3255$

Holen, Ø. H. (2013). "Disentangling taste and toxicity in aposematic prey". In: Proceedings of the Royal Society B 28o, p. 20122588 . Dor: 10.1098/ rspb.2012. 2588.

Holen, Ø. H. and R. A. Johnstone (2004). "The evolution of mimicry under constraints". In: The American Naturalist 164.5, pp. 598-613. Dor: 10.1086/424972. 
Hossie, T. J., T. N. Sherratt, D. H. Janzen, and W. Hallwachs (2013). "An eyespot that "blinks": an open and shut case of eye mimicry in Eumorpha caterpillars (Lepidoptera: Sphingidae)". In: Journal of Natural History 47-45-46, pp. 29152926. DOr: $10 \cdot 1080 / 00222933 \cdot 2013 \cdot 791935$.

Huheey, J. E. (1964). "Studies of warning coloration and mimicry. IV. A mathematical model of model-mimic frequencies". In: Ecology 45.1, pp. 185-188. DoI: $10.2307 / 1937125$

- (1976). "Studies in warning coloration and mimicry. VII. Evolutionary consequences of a Batesian-Müllerian spectrum: a model for Müllerian mimicry”. In: Evolution 30.1, pp. 86-93. Dor: 10.1111/ j .1558-5646.1976. tboo884 · .

Janzen, D. H., W. Hallwachs, and J. M. Burns (2010). “A tropical horde of counterfeit predator eyes". In: Proceedings of the National Academy of Sciences 107.26, pp. 11659-11665. Dor: 10.1073/pnas.0912122107.

Jaynes, E. T. (2003). Probability Theory: The Logic of Science. Ed. by G. L. Bretthorst. Cambridge University Press.

Kang, C., H. Moon, T. N. Sherratt, S.-I. Lee, and P. G. Jablonski (2016). "Multiple lines of anti-predator defence in the spotted lanternfly, Lycorma delicatula (Hemiptera: Fulgoridae)". In: Biological Journal of the Linnean Society 120.1, pp. 115-124. DOI: $10.1111 / \mathrm{bij} .12847$.

Kang, C., R. Zahiri, and T. N. Sherratt (2017). "Body size affects the evolution of hidden colour signals in moths". In: Proceedings of the Royal Society B 284.1861, p. 20171287 . DOI: $10.1098 / \mathrm{rspb} .2017 \cdot 1287$.

Kannan, D. (1983). "A Markov chain analysis of predator strategy in a modelmimic system". In: Bulletin of Mathematical Biology 45·3, pp. 347-400. Dor: 10 . 1016/Soog2-8240(83)80061-5. 
Kemp, C. and J. B. Tenenbaum (2009). "Structured statistical models of inductive reasoning". In: Psychological Review 116.1, pp. 20-58. Dor: 10.1037/a0014282.

Kokko, H., J. Mappes, and L. Lindström (2003). "Alternative prey can change model-mimic dynamics between parasitism and mutualism". In: Ecology Letters 6.12, pp. 1068-1076. Dor: $10.1046 / \mathrm{j} .1461-0248.2003 .00532 . \mathrm{x}$.

Krebs, J. R. and A. Kacelnik (1991). "Decision-making”. In: Behavioural Ecology: An Evolutionary Approach. Ed. by J. R. Krebs and N. B. Davies. 3rd ed. Oxford: Blackwell Scientific Publishing. Chap. 4, pp. 105-136.

Leimar, O., M. Enquist, and B. Sillén-Tullberg (1986). "Evolutionary stability of aposematic coloration and prey unprofitability: a theoretical analysis". In: The American Naturalist 128.4, pp. 469-49o. DoI: 10.1086/284581.

Leimar, O., B. S. Tullberg, and J. Mallet (2012). "Mimicry, saltational evolution, and the crossing of fitness valleys". In: The Adaptive Landscape in Evolutionary Biology. Ed. by E. I. Svensson and R. Calsbeek. Oxford University Press. Chap. 16, pp. 259-270.

Lewis, D. K. (1980). "A subjectivist's guide to objective chance". In: Studies in Inductive Logic and Probability. Ed. by R. C. Jeffrey. Vol. 2. Berkeley: University of California Press, pp. 263-293.

Liddell, H. G. and R. Scott (1940). A Greek-English Lexicon. Ed. by H. Stuart Jones and R. McKenzie. Oxford: Clarendon Press.

Luedeman, J. K., F. R. McMorris, and D. D. Warner (1981). "Predators encountering a model-mimic system with alternative prey". In: The American Naturalist 117.6, pp. 1040-1048. DoI: $10.1086 / 283794$.

MacDougall, A. and M. S. Dawkins (1998). "Predator discrimination error and the benefits of Müllerian mimicry". In: Animal Behaviour 55.5, pp. 1281-1288. DoI: 10.1006/anbe . 1997.0702 
Maldonado, H. (1970). "The deimatic reaction in the praying mantis Stagmatoptera biocellata". In: Zeitschrift für vergleichende Physiologie 68.1, pp. 6o-71.

Mallet, J. and M. Joron (1999). "Evolution of diversity in warning color and mimicry: polymorphisms, shifting balance, and speciation". In: Annual Review of Ecology and Systematics 30.1, pp. 201-233. Dor: $10.1146 /$ annurev . ecolsys . 30.1 .201 .

Marcum, J. I. (1947). A Statistical Theory of Target Detection by Pulsed Radar. Research rep. RM-754-PR. Santa Monica: RAND Corporation.

Marshall, G. A. K. (1908). “On diaposematism, with reference to some limitations of the Müllerian hypothesis of mimicry". In: Transactions of the Royal Entomological Society of London, pp. 93-142.

Matessi, C. and R. Cori (1972). "Models of population genetics of batesian mimicry". In: Theoretical Population Biology 3.1, pp. 41-68. Dor: 10 . 1016/0040$5809(72) 90033^{-0}$.

McGuire, L., H. Van Gossum, K. Beirinckx, and T. N. Sherratt (2006). "An empirical test of signal detection theory as it applies to Batesian mimicry". In: Behavioural Processes 73.3, pp. 299-307. Dor: 10.1016/ j . beproc.2006.07.004. Müller, F. (1879). "Ituna and Thyridia: a remarkable case of mimicry in butterflies". In: Proceedings of the Entomological Society of London, pp. $\mathrm{xx}-\mathrm{xxix}$.

Nicholson, A. J. (1927). "Presidential address: a new theory of mimicry in insects". In: Australian Zoologist 5, pp. 10-104.

Olofsson, M., H. Løvlie, J. Tibblin, S. Jakobsson, and C. Wiklund (2012). "Eyespot display in the peacock butterfly triggers antipredator behaviors in naïve adult fowl". In: Behavioral Ecology 24.1, pp. 305-310. Dor: 10.1093/beheco/ars 167 . 
Owen, R. E. and A. Owen (1984). "Mathematical paradigms for mimicry: recurrent sampling". In: Journal of Theoretical Biology 109.2, pp. 217-247. Dor: 10.1016/ So022-5193(84)80004-1.

Palmer, J., A. C. Huk, and M. N. Shadlen (2005). "The effect of stimulus strength on the speed and accuracy of a perceptual decision". In: Journal of Vision 5.5, p. 1. DOI: $10 \cdot 1167 / 5 \cdot 5 \cdot 1$.

Poulton, E. B. (1890). The Colours of Animals: Their Meaning and Use Especially Considered in the Case of Insects. London: Kegan Paul, Trench \& Trübner.

Punnett, R. C. (1915). Mimicry in Butterflies. Cambridge: Cambridge University Press.

Ruxton, G. D., W. L. Allen, T. N. Sherratt, and M. P. Speed (2018). Avoiding Attack: The Evolutionary Ecology of Crypsis, Warning Signals and Mimicry. 2nd ed. Oxford: Oxford University Press.

Ruxton, G. D., D. W. Franks, A. C. V. Balogh, and O. Leimar (2008). "Evolutionary implications of the form of predator generalization for aposematic signals and mimicry in prey". In: Evolution 62.11, pp. 2913-2921. Dor: $10.1111 / \mathrm{j} \cdot 1558-$ $5646.2008 .00485 \cdot x$.

Sanjana, N. E. and J. B. Tenenbaum (2003). "Bayesian models of inductive generalization". In: Advances in Neural Information Processing Systems. Ed. by S. Becker, S. Thrun, and K. Obermayer. Vol. 15. MIT Press, pp. 51-58.

Sasaki, A., I. Kawaguchi, and A. Yoshimori (2002). "Spatial mosaic and interfacial dynamics in a müllerian mimicry system". In: Theoretical Population Biology 61.1, pp. 49-71. Dor: $10.1006 / \mathrm{tpbi} .2001 .1552$.

Scholtyssek, C., D. C. Osorio, and R. J. Baddeley (2016). "Color generalization across hue and saturation in chicks described by a simple (Bayesian) model". In: Journal of Vision 16.10, p. 8. Dor: $10.1167 / 16.10 .8$ 
Shepard, R. N. (1987). "Toward a universal law of generalization for psychological science". In: Science 237.4820, pp. 1317-1323.

Sherratt, T. N. (2003). "State-dependent risk-taking by predators in systems with defended prey". In: OIKOS 103.1, pp. 93-100. Dor: $10.1034 /$ j . 1600-0706. $2003 \cdot 12576 x$.

- (2011). "The optimal sampling strategy for unfamiliar prey". In: Evolution 65.7, pp. 2014-2025. DOI: $10.1111 /$ j .1558-5646.2011.01274.x.

Sherratt, T. N. and C. A. Peet-Paré (2017). "The perfection of mimicry: an information approach". In: Philosophical Transactions of the Royal Society B 372.1724, p. 2016034 O. DOI: $10.1098 /$ rstb.2016.0340.

Sherratt, T. N., M. P. Speed, and G. D. Ruxton (2004). "Natural selection on unpalatable species imposed by state-dependent foraging behaviour". In: Journal of Theoretical Biology 228.2, pp. 217-226. Dor: 10.1016/ j . j tbi .2003.12.009.

Skelhorn, J., C. G. Halpin, and C. Rowe (2016). "Learning about aposematic prey". In: Behavioral Ecology 27.4, pp. 955-964. Dor: 10.1093/beheco/arwoog.

Skelhorn, J., G. G. Holmes, T. J. Hossie, and T. N. Sherratt (2016). "Eyespots”. In: Current Biology 26.2, R52-R54. Dor: 10.1016/ j .cub.2015.10.024.

Skelhorn, J., G. G. Holmes, and C. Rowe (2016). "Deimatic or aposematic?" In: Animal Behaviour 113, e1-e3. Dor: 10.1016/ j . anbehav.2015.07.021.

Skelhorn, J. and C. Rowe (2009). "Distastefulness as an antipredator defence strategy". In: Animal Behaviour 78.3, pp. 761-766. Dor: 10.1016/ j . anbehav.2009. 07.006 .

Skyrms, B. and J. A. Barrett (2019). "Propositional content in signals". In: Studies in History and Philosophy of Science Part C 74, pp. 34-39. Dor: $10.1016 / \mathrm{j}$. Shpsc.2019.01.005. 
Speed, M. P. (1993). "Muellerian mimicry and the psychology of predation". In: Animal Behaviour 45.3, pp. 571-580. Dor: 10.1006/anbe.1993.1067.

- (1999). "Batesian, quasi-Batesian or Müllerian mimicry? Theory and data in mimicry research". In: Evolutionary Ecology 13.7, pp. 755-776. Dor: 10.1023/A: 1010871106763 .

Speed, M. P. and J. R. G. Turner (1999). "Learning and memory in mimicry: II. Do we understand the mimicry spectrum?" In: Biological Journal of the Linnean Society 67.3, pp. 281-312. Dor: $10.1111 /$ j . 1095-8312.1999.tbo1935 . x.

Spence, K. W. (1937). "The differential response in animals to stimuli varying within a single dimension." In: Psychological Review 44.5, pp. 430-444. DoI: $10.1037 /$ hoo62885.

Spetch, M. L. and K. Cheng (1998). “A step function in pigeons' temporal generalization in the peak shift task". In: Animal Learning E Behavior 26.1, pp. 103118. DOr: $10.3758 / \mathrm{BF} 03199165$.

Staddon, J. E. R. (1975). "A note on the evolutionary significance of 'supernormal' stimuli". In: The American Naturalist 109.969, pp. 541-545. Dor: 10.1086/ 283025 .

Staddon, J. E. R. and A. K. Reid (1990). "On the dynamics of generalization". In: Psychological Review 97.4, pp. 576-578.

Staudinger, M. D., R. T. Hanlon, and F. Juanes (2011). "Primary and secondary defences of squid to cruising and ambush fish predators: variable tactics and their survival value". In: Animal Behaviour 81.3, pp. 585-594. Dor: 10.1016/ j. anbehav.2010.12.002

Stevens, M., A. Cantor, J. Graham, and I. S. Winney (2009). "The function of animal 'eyespots': conspicuousness but not eye mimicry is key". In: Current Zoology 55.5, pp. 319-326. 
Stevens, M. and G. D. Ruxton (2014). “Do animal eyespots really mimic eyes?” In: Current Zoology 6o.1, pp. 26-36. Dor: 10.1093/czoolo/6o.1.26.

Tenenbaum, J. B. and T. L. Griffiths (2001). "Generalization, similarity, and Bayesian inference". In: Behavioral and Brain Science 24, pp. 629-640.

Thorogood, R., H. Kokko, and J. Mappes (2017). "Social transmission of avoidance among predators facilitates the spread of novel prey". In: Nature Ecology \& Evolution 2.2, pp. 254-261. DOr: 10.1038/s41559-017-0418-x.

Turner, J. R. G. (1984). "Mimicry: the palatability spectrum and its consequences". In: The Biology of Butterflies. Ed. by R. I. Vane-Wright and P. Ackery. Princeton: Princeton University Press. Chap. 14, pp. 141-161.

Turner, J. R. G., E. P. Kearney, and L. S. Exton (1984). "Mimicry and the Monte Carlo predator: the palatability spectrum, and the origins of mimicry". In: Biological Journal of the Linnean Society 23, pp. 247-268.

Umbers, K. D. L., S. De Bona, T. E. White, J. Lehtonen, J. Mappes, and J. A. Endler (2017). "Deimatism: a neglected component of antipredator defence". In: Biology Letters 13.4, p. 20160936. Dor: 10.1098/rsbl.2016.0936.

Umbers, K. D. L. and J. Mappes (2015). "Postattack deimatic display in the mountain katydid, Acripeza reticulata". In: Animal Behaviour 100, pp. 68-73. Dor: $10.1016 / \mathrm{j}$.anbehav.2014.11.009.

Wiley, R. H. (1994). "Errors, exaggeration, and detection in animal communication". In: Behavioral Mechanisms in Evolutionary Ecology. Ed. by L. A. Real. Chicago: University of Chicago Press. Chap. 7, pp. 157-189.

Williamson, G. B. and C. E. Nelson (1972). "Fitness set analysis of mimetic adaptive strategies". In: The American Naturalist 106.950, pp. 525-537. Dor: 10 . $1086 / 282792$. 
Yachi, S. and M. Higashi (1998). "The evolution of warning signals". In: Nature 394, pp. 882-884. Dor: $10.1038 / 29751$. 


\section{UNNECESSARY EXTENSIONS TO THE MIXTURE MODEL}

In this appendix, I discuss two possible extensions to the mixture models used in Section 2.3 adding asymmetric mixing coefficients, and variable numbers of components. I show that both these extensions are almost included in the case I

use in Section 2.3, and the additional generality is not needed. Throughout this appendix, I use the notation of Section 2.3 for the mixture model.

In Section 2.3, it was assumed that each component $f\left(y \mid \theta_{i}\right)$ makes the same contribution to the mixture, and therefore has mixture coefficient $1 / n$. This corresponds to the biological assumption that all the populations are equally abundant, which is obviously not reasonable. An extension would allow arbitrary mixing coefficients $p=\left(p_{1}, \ldots, p_{n}\right) \in \Delta_{n}$, where $\Delta_{n}$ is the $n$-simplex. One could also vary the number of components. This is arguably more plausible than fixing $n$ beforehand, since we don't expect the predator to know in advance how many populations of prey there are. In this appendix, I will incorporate both $n$ and $p$ as parameters of the model together: if I wanted to make just one of them a parameter and to fix the other, I could then make the prior distribution of the fixed one a distribution with all its mass on one point.

Making $n$ and $p$ parameters will require them to have priors $v(n)$ and $\kappa(p \mid n)$ respectively, and I will assume the priors are independent of the priors on the parameters $\theta_{i}$. Given the priors, the joint distribution of $n, p, y$, and the parameters 
$\theta_{i}$ is

$$
v(n) \kappa(p \mid n) \sum_{i=1}^{n}\left[p_{i} f\left(y \mid \theta_{i}\right) \prod_{j=1}^{n} \pi\left(\theta_{j}\right)\right] .
$$

Integrating out $n, p$, and the $\theta_{i}$ shows that the marginal distribution is again the same as that for a single component, and so the posterior after making the observation $y$ is

$$
v(n) \mathcal{K}(p \mid n) \sum_{i=1}^{n} \frac{p_{i} f\left(y \mid \theta_{i}\right) \prod_{j=1}^{n} \pi\left(\theta_{j}\right)}{m(y)}=v(n) \mathcal{K}(p \mid n) \sum_{i=1}^{n}\left[p_{i} p\left(\theta_{i} \mid y\right) \prod_{\substack{j=1 \\ j \neq i}}^{n} \pi\left(\theta_{j}\right)\right],
$$

so $y$ provides no information about the distribution of $n$ and $p$. The predictions of the model all depend on the posterior predictive distribution, which is

$$
\begin{array}{r}
\sum_{n=1}^{\infty} \int_{\Theta^{n} \times \Delta_{n}} \sum_{i=1}^{n}\left(p_{i} f\left(y^{\prime} \mid \theta_{i}\right)\right) v(n) \kappa(p \mid n) \sum_{i=1}^{n}\left(p_{i} p\left(\theta_{i} \mid y\right) \prod_{\substack{j=1 \\
j \neq i}}^{n} \pi\left(\theta_{j}\right)\right] d \theta_{1} \cdots d \theta_{n} d p= \\
\sum_{n=1}^{\infty} v(n) \sum_{i=1}^{n} \sum_{j=1}^{n} \int_{\Theta^{n} \times \Delta_{n}} \kappa(p \mid n) p_{i} p_{j} f\left(y^{\prime} \mid \theta_{i}\right) p\left(\theta_{i} \mid y\right) \prod_{\substack{k=1 \\
h \neq j}} \pi\left(\theta_{k}\right) d \theta_{1} \cdots d \theta_{n} d p= \\
\mathbb{E}_{\mathcal{L}}\left[\sum_{i=1}^{n} p_{i}^{2}\right] \int_{\Theta} f\left(y^{\prime} \mid \theta\right) p(\theta \mid y) d \theta+\mathbb{E}_{\mathcal{L}}\left[1-\sum_{i=1}^{n} p_{i}^{2}\right] m\left(y^{\prime}\right) .
\end{array}
$$

Thus the only difference from the posterior predictive distribution in Section 2.3 is that we have replaced the mixture coefficient of $1 / n$ in the posterior predictive distribution with a new coefficient derived from the joint distribution of $n$ and $p$, $v(n) \kappa(p \mid n)$. Extending the model does not allow the predator to learn anything about the distribution of $n$ or $p$. This extension is a slight generalization, in that $\mathbb{E}_{v \kappa}\left[\sum_{i=1}^{n} p_{i}^{2}\right]$ can take on any value between 0 and 1 , while $1 / n$ is restricted to reciprocals of integers; but this generality is unnecessary for the purposes of Section 2.3. particularly as I can only plot finitely many mixture coefficients in any case. 
APPENDIX B

SUPPLEMENTARY FIGURES AND TABLES TO CHAPTER 2 


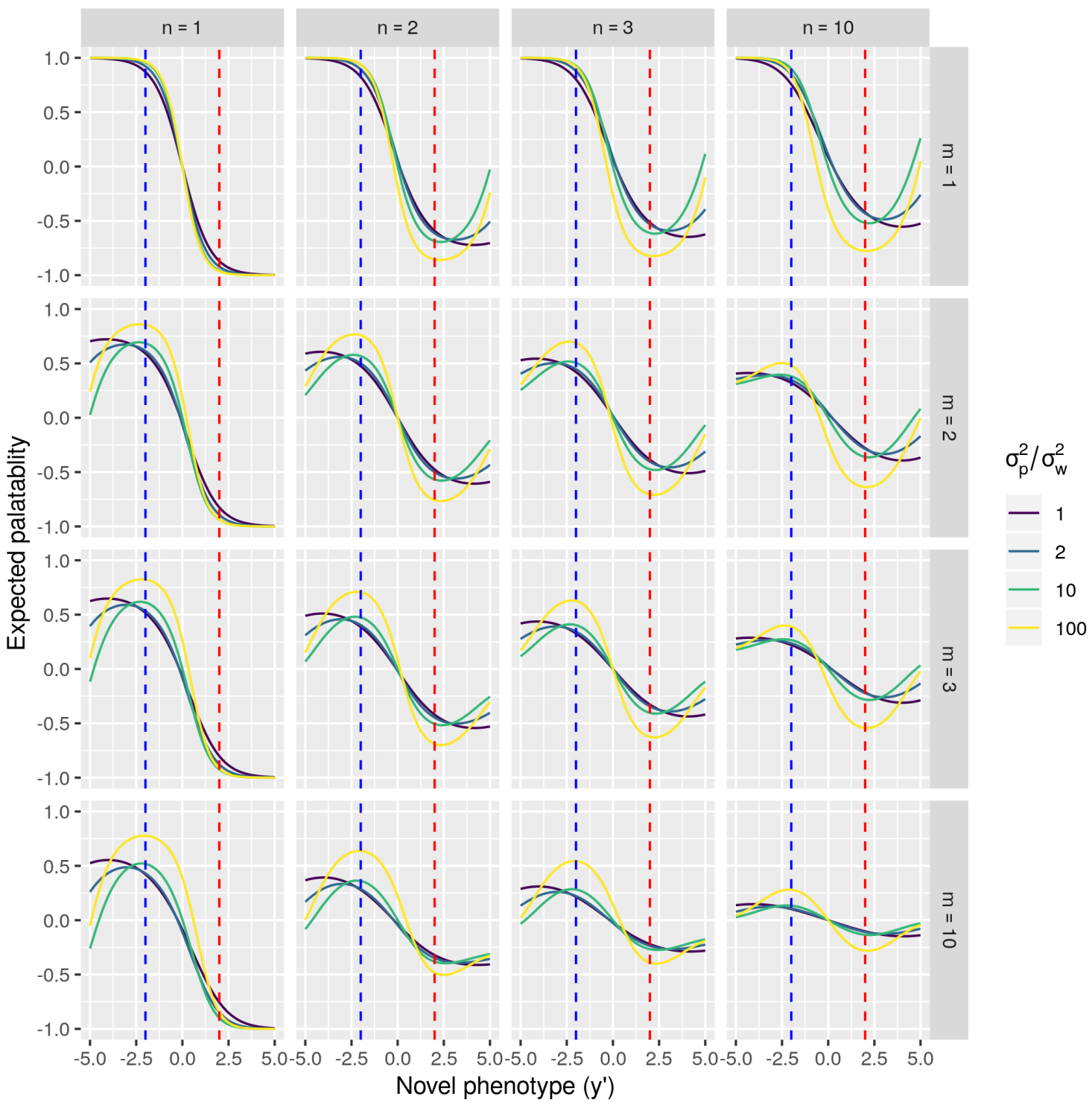

Figure B.1: Estimated palatabilities of a novel phenotype under a binary palatability model. $n$ and $m$ are the assumed number of populations of palatable and unpalatable prey respectively. The previous observations, palatable with phenotype -2 and unpalatable with phenotype 2 , are shown by the blue and red lines respectively. The phenotype is in units of $\sigma_{w}$. 


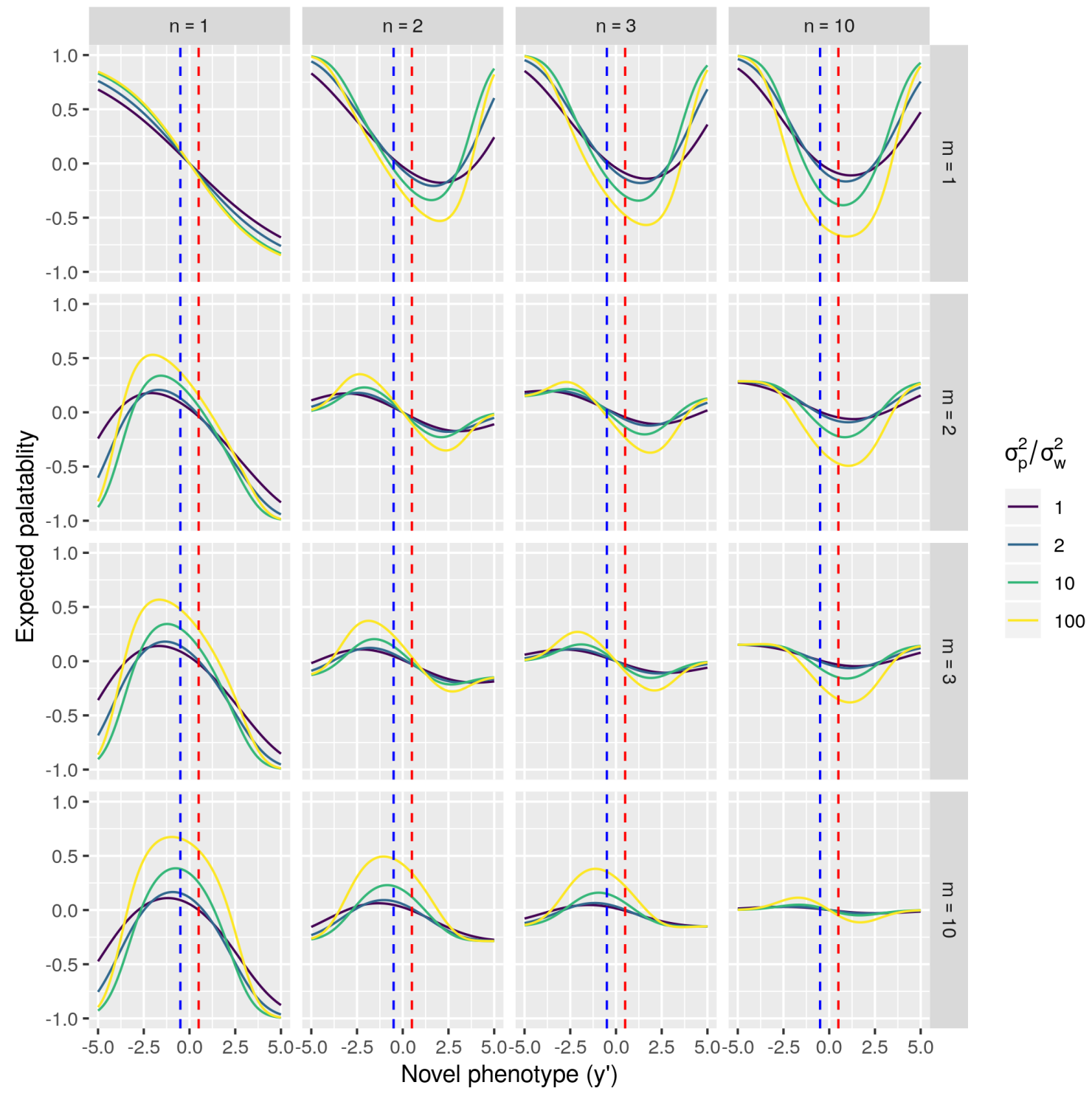

Figure B.2: Estimated palatabilities of a novel phenotype under a binary palatability model. $n$ and $m$ are the assumed number of populations of palatable and unpalatable prey respectively. The previous observations, palatable with phenotype -0.5 and unpalatable with phenotype 0.5 , are shown by the blue and red lines respectively. The phenotype is in units of $\sigma_{w}$. 
Table B.1: Degrees of peak shift from a binary palatability model. As Table 2.2 but fixing $\sigma_{p}^{2} / \sigma_{w}^{2}=10$, and with the predator having made observations at \pm the observation.

\begin{tabular}{|c|c|c|c|c|c|}
\hline$n$ & $m$ & Observation & Peak location & Peak shift & Peak value \\
\hline \multirow[t]{9}{*}{2} & \multirow[t]{3}{*}{2} & 0.5 & -2.11 & 1.61 & 0.230 \\
\hline & & 1.0 & -2.12 & 1.12 & 0.407 \\
\hline & & 2 & -2.38 & 0.383 & 0.578 \\
\hline & \multirow[t]{3}{*}{3} & 0.5 & -1.56 & 1.06 & 0.203 \\
\hline & & 1.0 & -1.81 & 0.805 & 0.340 \\
\hline & & 2 & -2.31 & 0.306 & 0.481 \\
\hline & \multirow[t]{3}{*}{10} & 0.5 & -0.85 & 0.348 & 0.230 \\
\hline & & 1.0 & -1.34 & 0.344 & 0.285 \\
\hline & & 2 & -2.23 & 0.227 & 0.364 \\
\hline \multirow[t]{9}{*}{3} & \multirow[t]{3}{*}{2} & 0.5 & -2.65 & 2.15 & 0.215 \\
\hline & & 1.0 & -2.31 & 1.31 & 0.360 \\
\hline & & 2 & -2.42 & 0.418 & 0.517 \\
\hline & \multirow[t]{3}{*}{3} & 0.5 & -1.90 & 1.40 & 0.155 \\
\hline & & 1.0 & -1.95 & 0.947 & 0.280 \\
\hline & & 2 & -2.33 & 0.328 & 0.411 \\
\hline & \multirow[t]{3}{*}{10} & 0.5 & -0.94 & 0.445 & 0.160 \\
\hline & & 1.0 & -1.40 & 0.404 & 0.213 \\
\hline & & 2 & -2.23 & 0.234 & 0.285 \\
\hline \multirow[t]{9}{*}{10} & \multirow[t]{3}{*}{2} & 0.5 & -5.07 & 4.57 & 0.286 \\
\hline & & 1.0 & -3.14 & 2.12 & 0.312 \\
\hline & & 2 & -2.60 & 0.595 & 0.396 \\
\hline & \multirow[t]{3}{*}{3} & 0.5 & -3.79 & 3.29 & 0.152 \\
\hline & & 1.0 & -2.62 & 1.62 & 0.195 \\
\hline & & 2 & -2.45 & 0.454 & 0.273 \\
\hline & \multirow[t]{3}{*}{10} & 0.5 & -1.66 & 1.16 & 0.049 \\
\hline & & 1.0 & -1.75 & 0.746 & 0.090 \\
\hline & & 2 & -2.28 & 0.277 & 0.136 \\
\hline
\end{tabular}




\section{UPPER BOUND ON $n^{*}$}

First note that $V_{0} \geq 0$ and $V_{0} \geq v_{d} P\left(D=1 \mid X_{0}\right)+v_{u} P\left(D=0 \mid X_{0}\right)$.

Since $P\left(\bar{X}_{n}>\lambda_{n} \mid X_{0}, D\right)$ is bounded by 0 and 1 ,

$$
V_{n} \leq v_{d} P\left(D=1 \mid X_{0}\right)(1-\Delta)^{n}+v_{u} P\left(D=0 \mid X_{0}\right)\left(1-(1-\Xi)^{n}\right) .
$$

Then

$$
\begin{aligned}
& n \geq-\frac{\log 2}{\log (1-\min (\Xi, \Delta))} \\
& n \log (1-\min (\Xi, \Delta)) \leq-\log 2 \\
&(1-\min (\Xi, \Delta))^{n} \leq \frac{1}{2} \\
&(1-\Xi)^{n} \leq \frac{1}{2} \quad \text { and } \quad(1-\Delta)^{n} \leq \frac{1}{2} \\
&(1-\Xi)^{n}+(1-\Delta)^{n} \leq 1 \\
&(1-\Xi)^{n}(1-\Delta)^{n} \leq 1-(1-\Xi)^{n}-(1-\Delta)^{n}+(1-\Xi)^{n}(1-\Delta)^{n} \\
&=\left(1-(1-\Xi)^{n}\right)\left(1-(1-\Delta)^{n}\right) \\
& \frac{(1-\Xi)^{n}(1-\Delta)^{n}}{\left(1-(1-\Xi)^{n}\right)\left(1-(1-\Delta)^{n}\right)} \leq 1 \\
&=\frac{(1-\Xi)^{n}\left(-v_{u}\right) P\left(D=0 \mid X_{0}\right) \quad(1-\Delta)^{n} v_{d} P\left(D=1 \mid X_{0}\right)}{(1-\Delta)^{n} v_{d} P\left(D=1 \mid X_{0}\right)} \frac{(1-\Xi)^{n}\left(-v_{u}\right) P\left(D=0 \mid X_{0}\right)}{}
\end{aligned}
$$


Then either

$$
\begin{aligned}
\frac{(1-\Xi)^{n}}{1-(1-\Xi)^{n}} & \leq \frac{(1-\Xi)^{n}\left(-v_{u}\right) P\left(D=0 \mid X_{0}\right)}{(1-\Delta)^{n} v_{d} P\left(D=1 \mid X_{0}\right)} \\
\frac{(1-\Delta)^{n}}{1-(1-\Xi)^{n}} & \leq \frac{\left(-v_{u}\right) P\left(D=0 \mid X_{0}\right)}{v_{d} P\left(D=1 \mid X_{0}\right)} \\
v_{d} P\left(D=1 \mid X_{0}\right)(1-\Delta)^{n} & \leq-v_{u} P\left(D=0 \mid X_{0}\right)\left(1-(1-\Xi)^{n}\right) \\
v_{d} P\left(D=1 \mid X_{0}\right)(1-\Delta)^{n}+ & \\
v_{u} P\left(D=0 \mid X_{0}\right)\left(1-(1-\Xi)^{n}\right) & \leq 0 \\
V_{n} & \leq V_{0},
\end{aligned}
$$

or

$$
\begin{gathered}
\frac{(1-\Delta)^{n}}{1-(1-\Delta)^{n}} \leq \frac{(1-\Delta)^{n} v_{d} P\left(D=1 \mid X_{0}\right)}{(1-\Xi)^{n}\left(-v_{u}\right) P\left(D=0 \mid X_{0}\right)} \\
\frac{(1-\Xi)^{n}}{1-(1-\Delta)^{n}} \leq \frac{v_{d} P\left(D=1 \mid X_{0}\right)}{\left(-v_{u}\right) P\left(D=0 \mid X_{0}\right)} \\
-v_{u} P\left(D=0 \mid X_{0}\right)(1-\Xi)^{n} \leq v_{d} P\left(D=1 \mid X_{0}\right)\left(1-(1-\Delta)^{n}\right) \\
-v_{u} P\left(D=0 \mid X_{0}\right)+ \\
v_{u} P\left(D=0 \mid X_{0}\right)\left(1-(1-\Xi)^{n}\right) \leq v_{d} P\left(D=1 \mid X_{0}\right)-v_{d} P\left(D=1 \mid X_{0}\right)(1-\Delta)^{n} \\
v_{d} P\left(D=1 \mid X_{0}\right)(1-\Delta)^{n}+ \\
v_{u} P\left(D=0 \mid X_{0}\right)\left(1-(1-\Xi)^{n}\right) \leq v_{d} P\left(D=1 \mid X_{0}\right)+v_{u} P\left(D=0 \mid X_{0}\right) \\
V_{n} \leq V_{0} .
\end{gathered}
$$


APPENDIX D

SUPPLEMENTARY FIGURES TO CHAPTER 3 


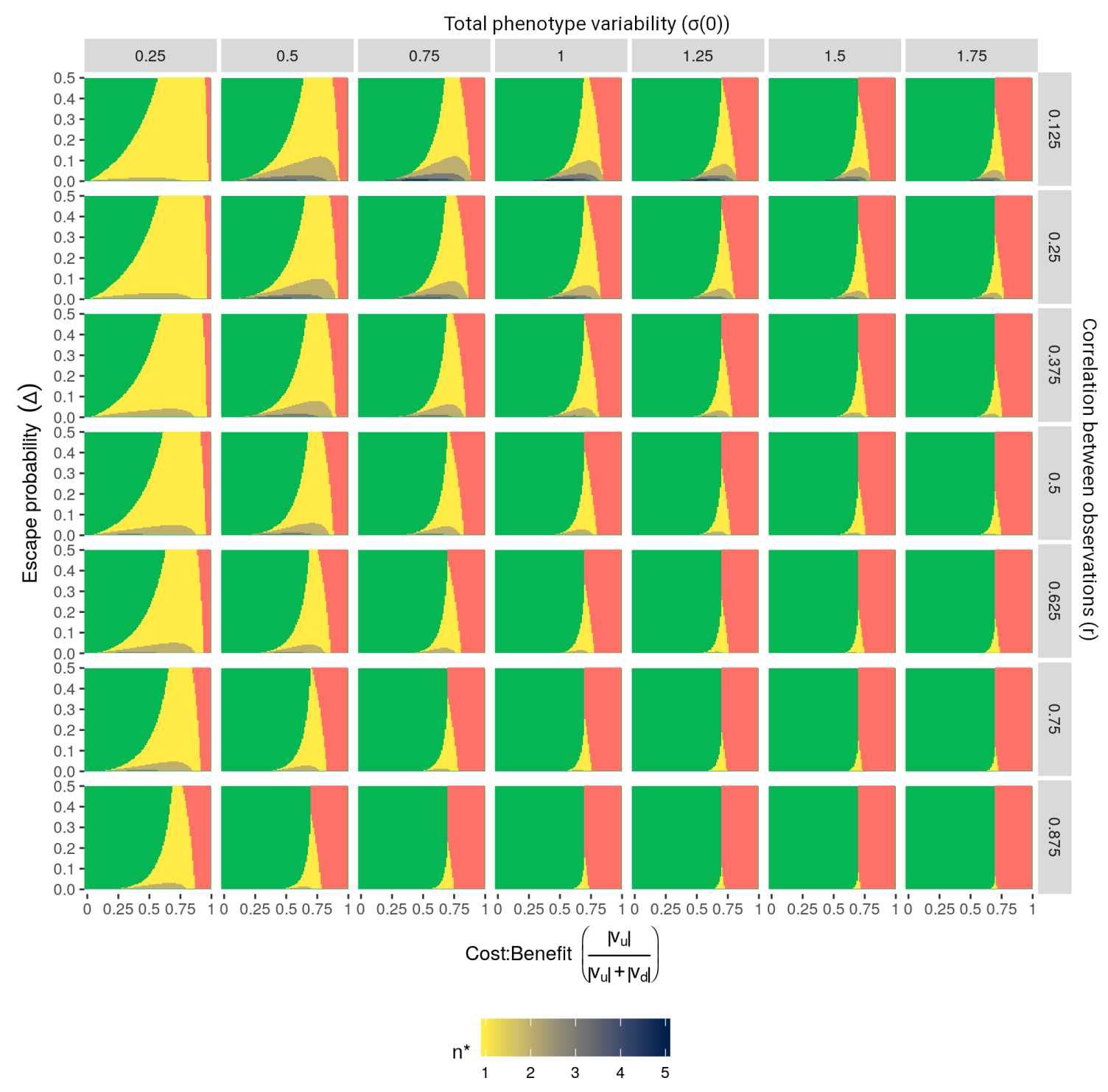

Figure D.1: The optimal action after having made a single observation of the prey. As Figure 3.2 , but with $\Xi=0.05$ and $\Delta$ on the y-axis. 


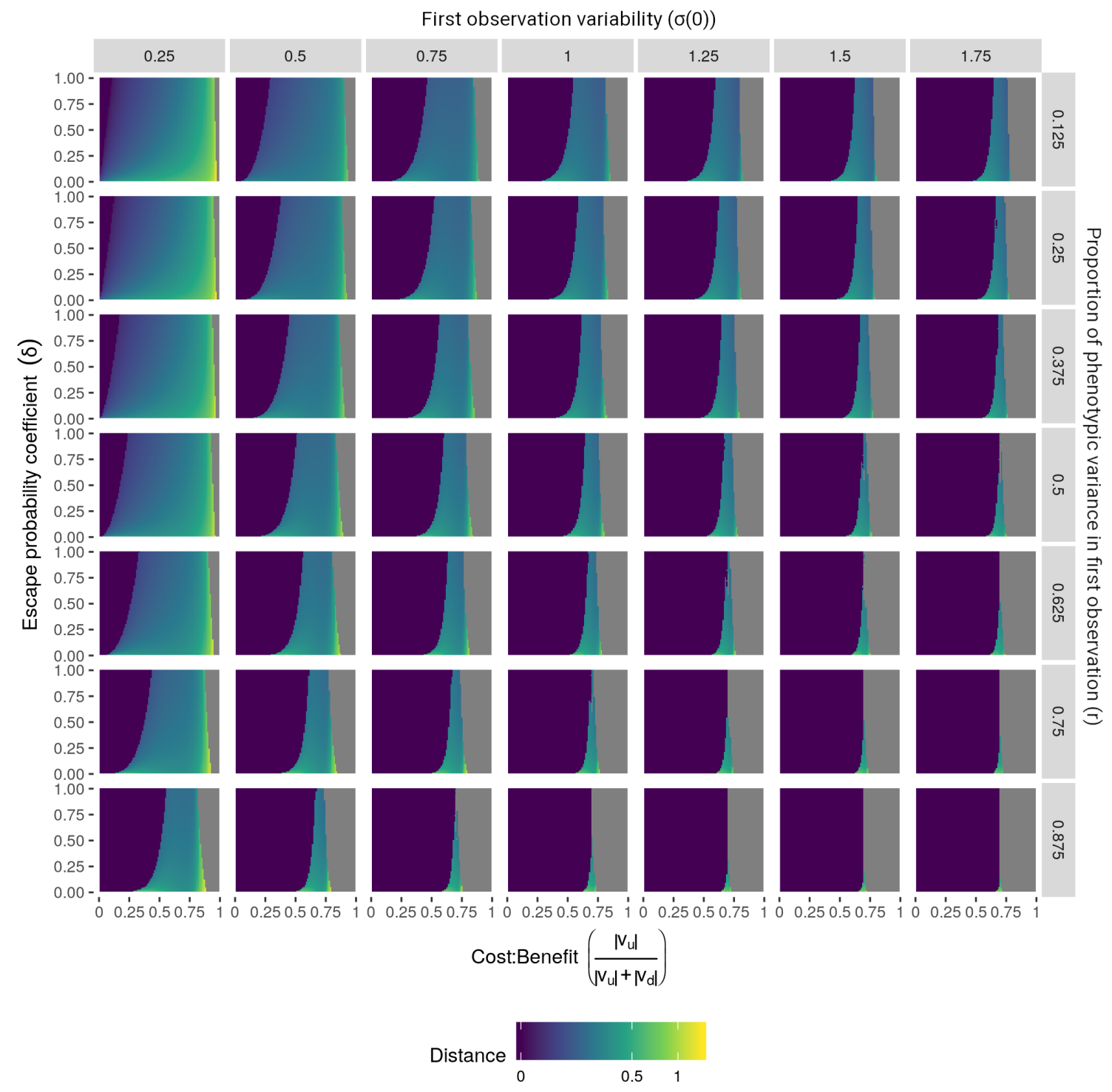

Figure D.2: The optimal distance $d$ to make a single additional observation after having made an observation at a distance of 1 . As Figure 3.4 , but with $\xi=20$ and $\delta$ on the y-axis. 


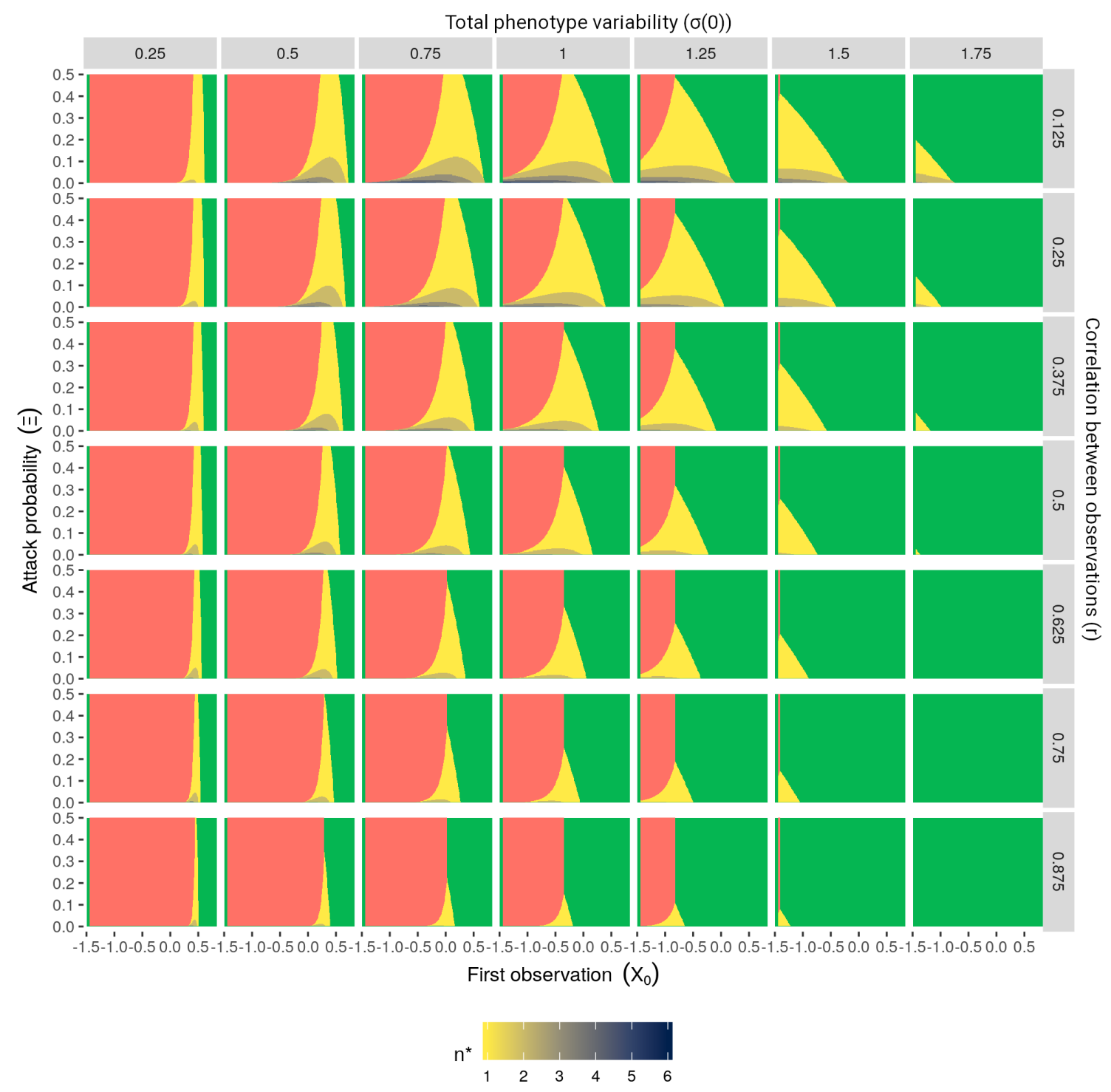

Figure D.3: The optimal action after having made a single observation of the prey. As Figure 3.1. but with $\Xi$ on the y-axis and $\left|v_{u}\right|=\left|v_{d}\right|$. 


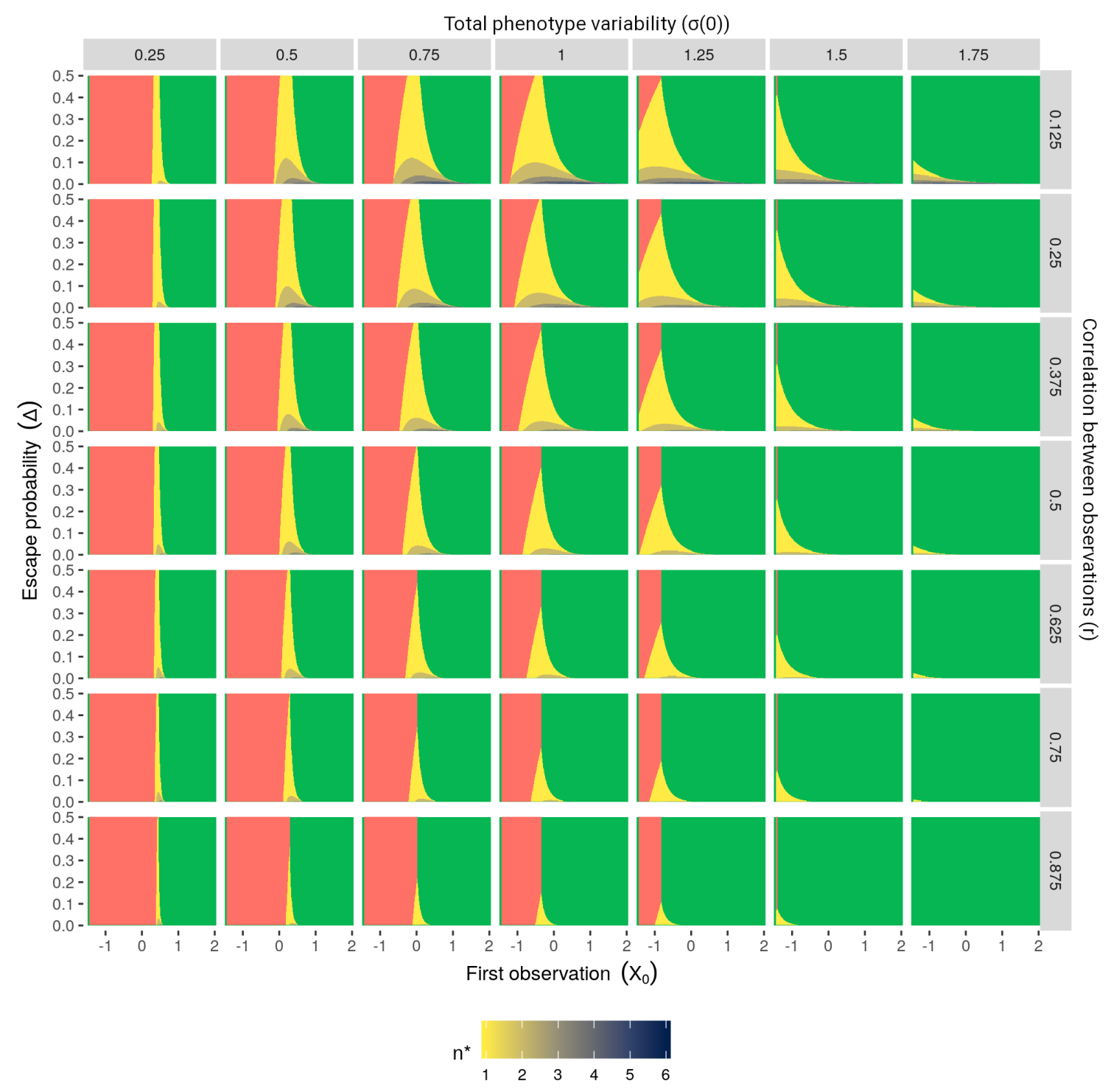

Figure D.4: The optimal action after having made a single observation of the prey. As Figure 3.1, but with $\Delta$ on the y-axis and $\left|v_{u}\right|=\left|v_{d}\right|$. 


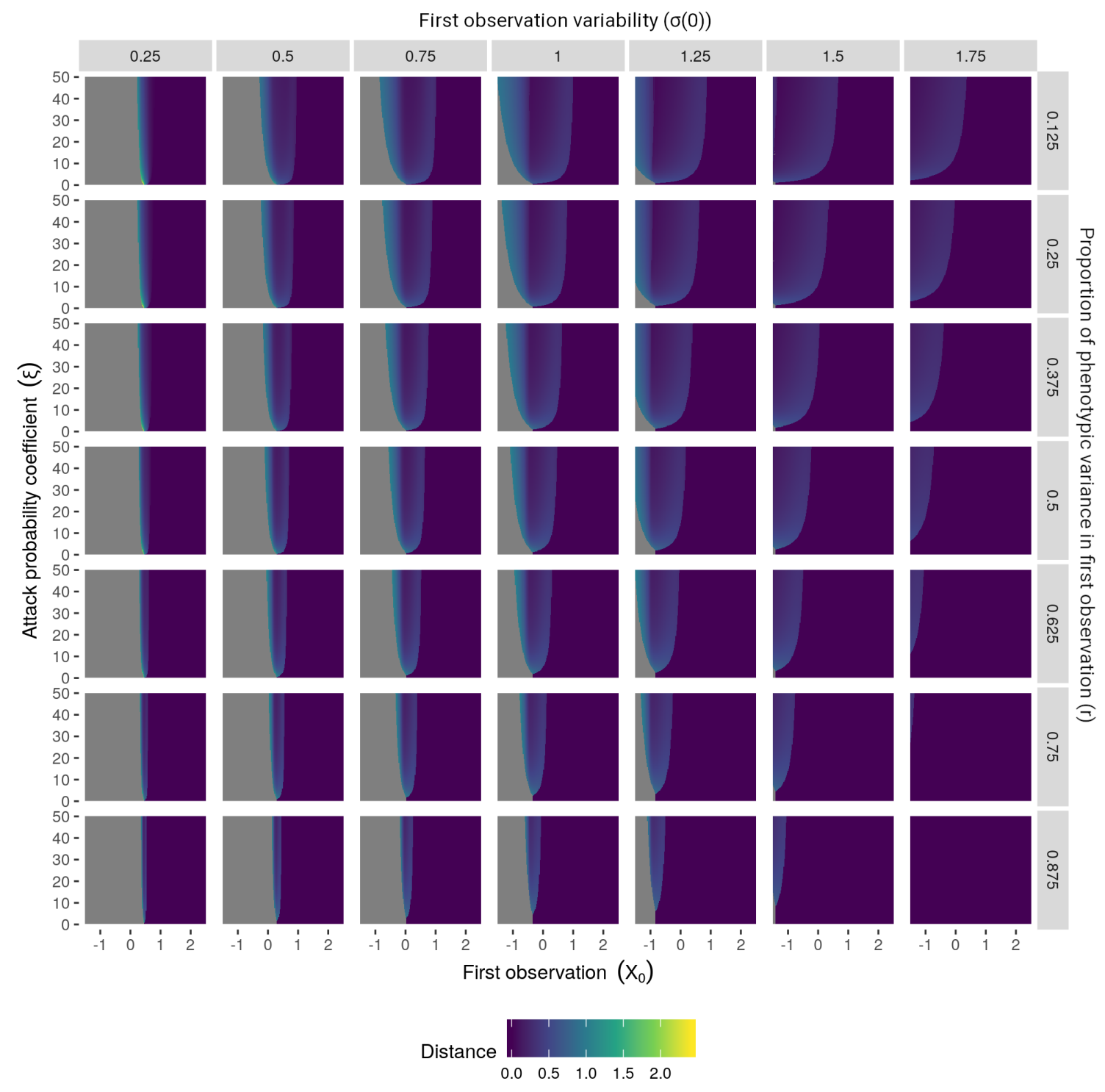

Figure D.5: The optimal action after having made a single observation of the prey. As Figure 3.3, but with $\xi$ on the y-axis and $\left|v_{u}\right|=\left|v_{d}\right|$. 


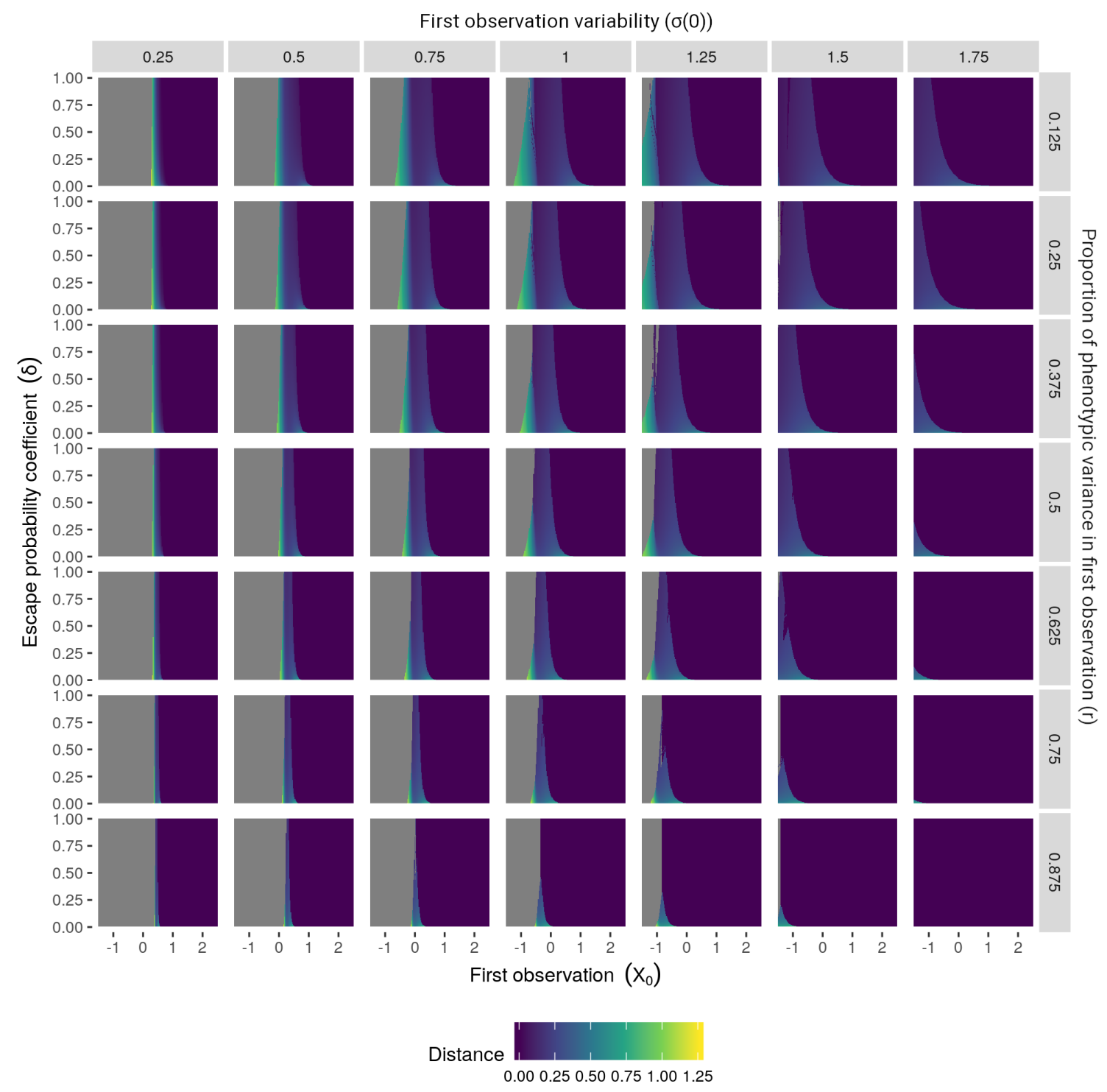

Figure D.6: The optimal action after having made a single observation of the prey. As Figure 3.1, but with $\delta$ on the $y$-axis and $\left|v_{u}\right|=\left|v_{d}\right|$. 


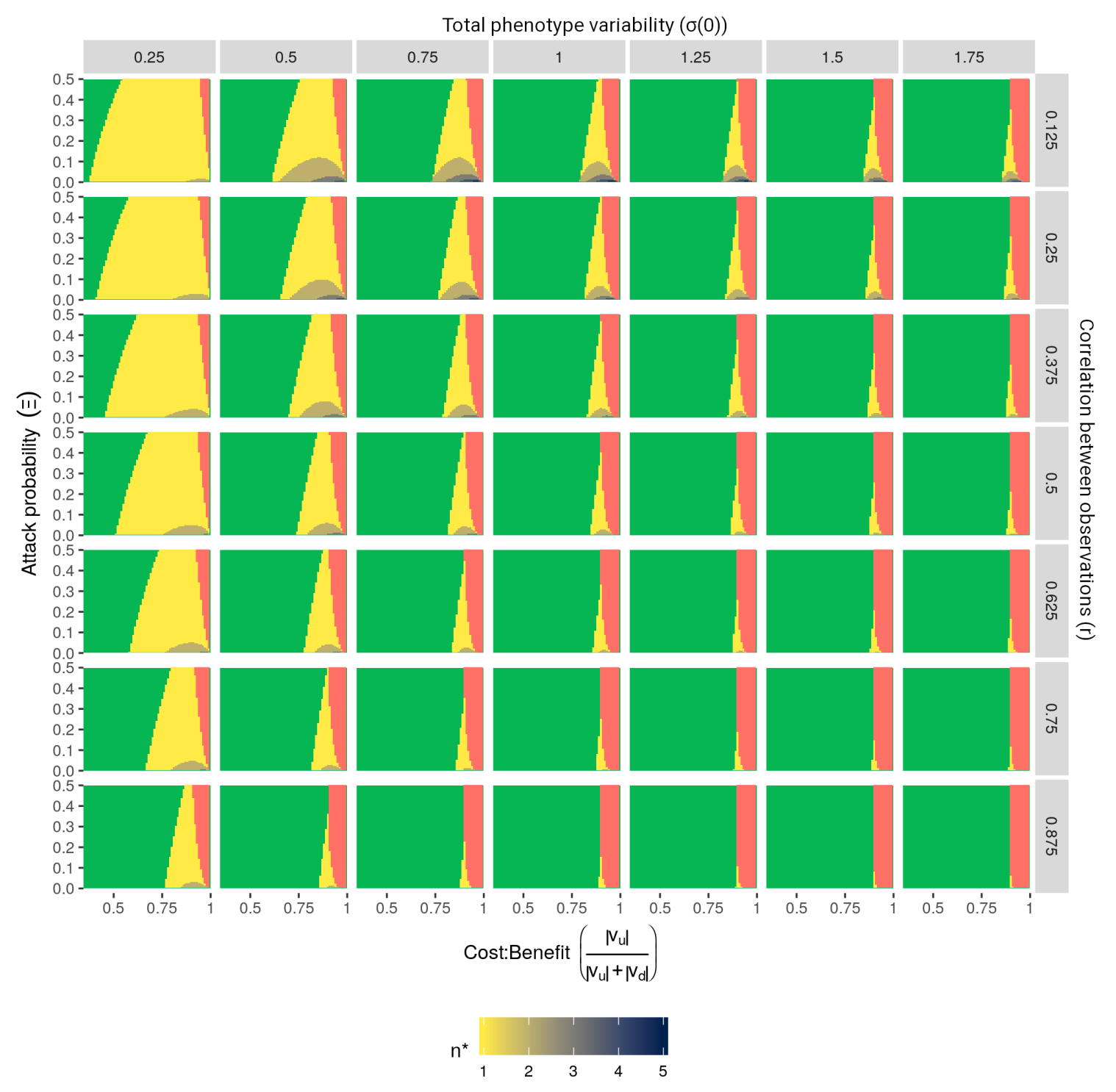

Figure D.7: The optimal action after having made a single observation of the prey. As Figure 3.2, but with $p=0.9$. 


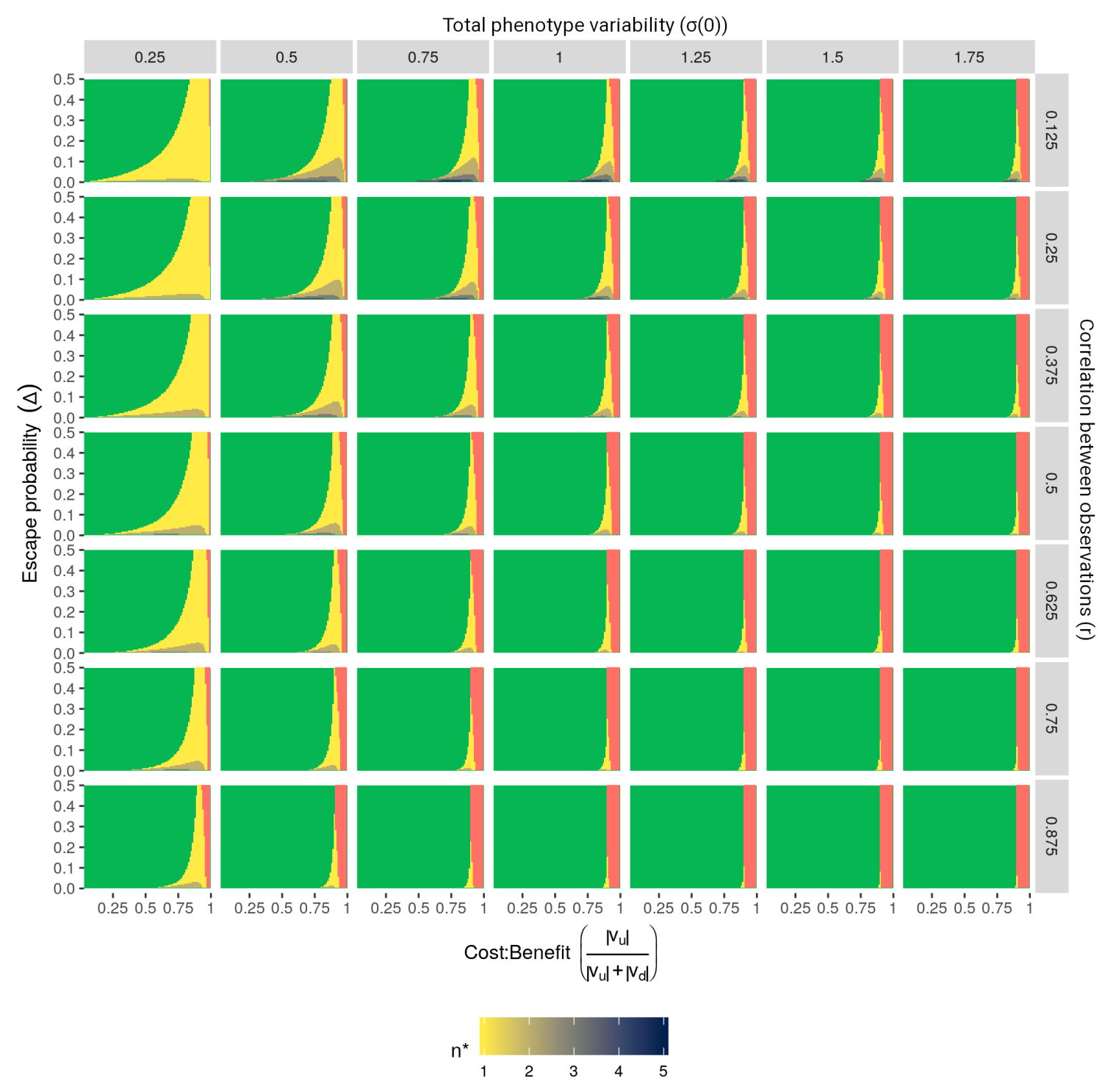

Figure D.8: The optimal action after having made a single observation of the prey. As Figure D.1. but with $p=0.9$. 